\title{
How to pare a pair: Topology control and pruning in intertwined complex networks
}

\author{
Felix Kramer $\oplus^{1,2, *}$ and Carl D. Modes $\circledast^{1,2,3, \dagger}$ \\ ${ }^{1}$ Max Planck Institute for Molecular Cell Biology and Genetics (MPI-CBG), Dresden 01307, Germany \\ ${ }^{2}$ Center for Systems Biology Dresden (CSBD), Dresden 01307, Germany \\ ${ }^{3}$ Cluster of Excellence, Physics of Life, TU Dresden, Dresden 01307, Germany
}

(Received 20 February 2020; accepted 1 October 2020; published 2 November 2020)

\begin{abstract}
Recent work on self-organized remodeling of vasculature in slime molds, leaf venation systems, and vessel systems in vertebrates has put forward a plethora of potential adaptation mechanisms. All these share the underlying hypothesis of a flow-driven machinery, meant to alter rudimentary vessel networks in order to optimize the system's dissipation, flow uniformity, or more, with different versions of constraints. Nevertheless, the influence of environmental factors on the long-term adaptation dynamics as well as the network structure and function have not been fully understood. Therefore, interwoven capillary systems such as those found in the liver, kidney, and pancreas present a novel challenge and key opportunity regarding the field of coupled distribution networks. We here present an advanced version of the discrete Hu-Cai model, coupling two spatial networks in three dimensions. We show that spatial coupling of two flow-adapting networks can control the onset of topological complexity in concert with short-term flow fluctuations. We find that both fluctuation-induced and spatial coupling induced topology transitions undergo curve collapse, obeying simple functional rescaling. Further, our approach results in an alternative form of Murray's law, which incorporates local vessel interactions and flow interactions. This geometric law allows for the estimation of the model parameters in ideal Kirchhoff networks and respective experimentally acquired network skeletons.
\end{abstract}

DOI: 10.1103/PhysRevResearch.2.043171

\section{INTRODUCTION}

Many recent studies on biological transportation networks have been focused on the hypothesis that vasculature is remodeled according to the flow-induced stress sensed by the cells making up the tissue [1]. This self-organized process optimizes the structures for the task at hand, e.g., distributing oxygen and nutrients, getting rid of waste, and carrying local secretion. The actual tissue response is dependent on the timescales probed. On the one hand, short-term changes usually concern rapid vessel diameter changes in response to pressure fluctuations or medication. On the other hand, longterm effects (e.g., due to metabolic changes) may manifest in permanent diameter changes [2], usually leaving the vessel structure with a trade-off between efficiency and redundancy [3].

Particular focus has been directed to the long-term remodeling of the capillary plexus and other rudimentary transport systems in the early developmental stages of organisms, i.e., by studying complex signaling cascades involving growth

\footnotetext{
*kramer@mpi-cbg.de

†modes@mpi-cbg.de
}

Published by the American Physical Society under the terms of the Creative Commons Attribution 4.0 International license. Further distribution of this work must maintain attribution to the author(s) and the published article's title, journal citation, and DOI. Open access publication funded by the Max Planck Society. factors like vascular endothelial growth factor in vascular systems of mammals [4] or auxin in plants [5]. Yet, the onset of refinement seems to be correlated with mechanical stresses (such as shear flow), as has been shown in a variety of model organisms from chicken embryos [1,6] and zebrafish [7] to leaves [8] and slime mold [9].

Early theoretical approaches by Murray [10,11] posited that diameter adaptation would minimize the overall power dissipation of the system. Following this ansatz of network optimization, many recent models used global optimization schemes on expanded vessel networks. These models captured the phenomenon of link pruning involving random damage, flow fluctuations, or rescaled volume costs and have been able to account for the trade-off of shunting and redundancies [12-14]. Further advances have been made in empirical studies of local vessel dynamics, e.g., blood vessel systems [15-17]. Local adaptation dynamics were also effectively derived by minimizing various effective network costs via gradient descent methods $[18,19]$. It has further been shown that the outcomes of locally adapting networks are robust against variations of the initial topological structure [20] and that plexus growth and correlated flow fluctuations can provide elaborate hierarchies [3,21]. Many of these effects may also be seen in continuous adaptation models in porous media $[22,23]$. It is interesting and important to note here that these adaptation mechanisms may leave certain fingerprints, e.g., in the form of allometric [24] and geometric laws [25].

These studies typically involve volume or metabolic constraints applied to abstract Kirchhoff networks. Yet they disregard the key characteristic common to all fluid transport 
systems: spatial embedding, which matters especially in the case of capillary systems as these directly interact via transport of metabolites with the surrounding tissue. These systems have to maintain a robust structure while being embedded in a possibly stiff tissue environment, potentially perturbing the shear stress driven adaptation mechanism.

We here focus on the development and function of multicomponent flow networks, which influence each other based on their spatial architecture. Biologically speaking, these systems often consist primarily of blood vessels and a secondary entangled, interacting system as found, for example, in liver lobule [26-29], kidney nephrons [30,31], pancreas [32-34], and lymphatic system [35]. Additionally, we intend to include the phenomenon of one flow network being caged by another complementary structure, e.g., capillaries embedded in bone marrow [36].

In this work, we study the adaptation of two coupled spatial networks according to an advanced version of the discrete Hu-Cai model [18], including Corson fluctuations [14]. Each network is subject to flow-driven and volume-constrained optimization on its own. Meanwhile, we introduce the networks' interaction in the form of a mutual repulsion or attraction of vessel surfaces. Repulsion will prevent them from touching directly by their otherwise flow-driven radius expansion, introducing a competition of the two networks for the space they are embedded in. Alternatively, vessel surfaces could be be attracted toward each other, presenting a positive feedback toward maintaining intertwined structures. In combination with fluctuation-induced redundancy, we find mutual repulsion to greatly reduce the networks' relative loop density even when strong fluctuations are present. On the other hand, we observe the emergence of a sharp transition toward a loopy state when there is attraction between the two networks.

Unfortunately, few metrics provide the means to fit or estimate the applied parameters of adaptation models for real systems, even though time-lapse experiments [7], counting pruning events, and topology analysis on pruned structures $[3,37,38]$ allow for qualitative insights into the mechanism at hand for certain model organisms. Yet there has been no proposal to our knowledge to quantitatively acquire or fit the model parameters from real, pruned network structures. In particular, interwoven systems present a special challenge as typical experimental setups for a full three dimensional (3D) reconstruction involve invasive measures, i.e., sacrificing the specimen and preventing any long-term vessel observation. To tackle this problem, we generalize an important scaling law, which has been discussed again recently in this context [39]: Murray's law. Our generalization enables us to reconstruct the model parameters with high fidelity for Kirchhoff networks solely from a given graph topology and it's edge radii distribution (assuming the pruning process reached a stationary state). We find order of magnitude estimate for these parameters in experimentally acquired data sets of an interwoven system: sinusoids and canaliculi in the mouse's liver acinus.

We begin our study in Sec. II with a brief reminder of the hydrodynamical and network theoretical background on which we operate. Next, we set up our model framework and its crucial components in detail. In Sec. III, we present the numerical evaluation of our model, in particular illuminating the cases of repulsive and attractive coupling between networks.
In Sec. IV, we derive and test our generalized geometric laws on ideal Kirchhoff networks and on data sets of vessel networks provided by our collaborators. We then go on to discuss the implications and limits of our model framework in the concluding section, Sec. V.

\section{THEORETICAL FRAMEWORK}

The following subsections are intended to provide the reader with the necessary background to proceed to the complex adaptation dynamics on ramified vascular networks that follow. Readers familiar with the general formalities should feel free to skip ahead to Sec. IIC, where we discuss our general setup.

First, we introduce the framework of Kirchhoff networks, as these provide us with the mathematical tools needed to describe complex flow landscapes. Afterward, we reintroduce the cost-function ansatz, the associated metabolic costs, and the chosen method of optimization which will render toward adaptation dynamics. Next, we discuss the intended hydrodynamic regime and the geometry of the intertwined system. We then extend our established framework by including fluctuation of the flow landscape as an essential tool to generate robust distribution networks. Finally, in the last subsection we introduce the relevant order parameters and metrics.

\section{A. Fundamentals of linear networks}

We model the biological vessel networks of interest as a composition of $m$ edges and $n$ vertices (branching points). Each edge carries a flux $f$ such that at any vertex the sum of all currents equal a nodal function $s$,

$$
\sum_{e \in \operatorname{inc}(v)} f_{e}=s_{v},
$$

where inc $(n)$ indicates the set of edges incident to vertex $v$. We refer to $s$ as sink or source when $s$ is nonzero. Equation (1) is Kirchhoff's current law, which represents mass conversation at every vertex. Further, in linear flow networks one may formulate the flux as a linear function, Ohm's law, as

$$
f_{e}=c_{e} \Delta p_{e},
$$

where $c_{e}$ is the conductance of an edge and $\Delta p_{e}$ its respective potential gradient. One may thus characterize the flux in every vessel as a direct response to a gradient of potential energy, concentration, temperature, etc., and have it scale linearly with the conductance which incorporates the geometry and physical nature of the transport problem. The equation systems formulated in (1) and (2) may be bundled in vectorial notation as

$$
\begin{gathered}
B \cdot f=s, \\
f=C \cdot \Delta p .
\end{gathered}
$$

Here $\boldsymbol{B}$ designates the incidence matrix and $\boldsymbol{C}$ is a diagonal matrix with $c_{e}$ on the diagonal. Combining Eqs. (3) and (4), one finds the transformation between the sinksor sources and the potentials as

$$
\boldsymbol{B} \cdot \boldsymbol{C} \cdot \Delta \boldsymbol{p}=\boldsymbol{s}
$$


The potential gradients can be calculated from nodal potentials as $\Delta \boldsymbol{p}=\boldsymbol{B}^{T} \cdot \boldsymbol{p}$. Hence, one may solve Eq. (5) as

$$
\Delta \boldsymbol{p}=\boldsymbol{B}^{T}\left[\boldsymbol{B} \cdot \boldsymbol{C} \cdot \boldsymbol{B}^{T}\right]^{\dagger} \boldsymbol{s},
$$

where $[\cdot]^{\dagger}$ designates the generalized inverse [40]. Interestingly enough, one may find a unique solution to the problem (5) by applying the Thomson principle [41,42]. Following this variational principle, one considers the system to be characterized by a cost function, i.e., the energy dissipation defined as $\sum_{e} f_{e}^{2} / \tilde{c}_{e}$, with positive coefficients $\tilde{c}_{e}>0$. Further, one may use this cost to formulate an optimization problem with Lagrange multipliers $p_{v}$ and the boundary conditions (1) such that

$$
\Gamma=\sum_{e} f_{e}^{2} / \tilde{c}_{e}+\sum_{v} p_{v}\left(s_{v}-\sum_{e^{\prime} \in \operatorname{inc}(v)} f_{e^{\prime}}\right) .
$$

The aim is to find the set of flows $f_{e}$ which minimize the system's cost, $\Gamma$, with respect to the constraints given by the Kirchhoff current law. Doing so, one will end up naturally with Ohm's law, with the conductance $c_{e}=\tilde{c}_{e}$ for the coefficients and the Lagrange parameters $p_{v}$ representing the nodal potentials. These $p_{v}$ define the potential gradients as $\Delta p_{e}=$ $\left(p_{\omega(e)}-p_{\alpha(e)}\right)$, where $\alpha(e), \omega(e)$ designate the initial and final vertexes of any edge $e$. This cost-function ansatz enables one to find a unique solution for the potential differences $\Delta p$ in Eq. (5) as

$$
\Delta p=C^{-1 / 2}\left[B \cdot C^{1 / 2}\right]^{\dagger} s .
$$

This solution is equivalent to (6) and represents the optimal potential landscape, which minimizes the overall power dissipation for a given landscape of conductance and sinks $[43,44]$. Note that this formalism may be applied to any stationary transport process following the Thomson principle as well as to random walks of particles on a lattice. This class of systems is often referred to as lumped systems or Kirchhoff networks, in analogy to simple electric circuits [45].

\section{B. Cost-function ansatz and optimization of biological networks}

The concept of characterizing a transport network by a cost may readily be transferred to dynamic biological systems. The cells which are forming the walls of vascular networks, for example, are able to respond and adapt to a given set of stimuli such as shear stress or hydrostatic pressure. This enables such systems to continuously change their own topology and edge conductance in order to reach final refined structures. To capture this behavior, one may formulate a cost for a vessel systems as proposed in Ref. [12]:

$$
\Gamma=\sum_{e} \frac{f_{e}^{2}}{c_{e}}+a c_{e}^{\gamma},
$$

where the first term is the power dissipation as before and the second is a metabolic cost term $c_{e}^{\gamma}$, with proportionality factor $a$. This second term encapsulates the notion that a biological organism is constrained by the metabolic costs to deploy and sustain a vessel of a certain conductance. The exponent $\gamma$ represents a degree of freedom to vary the relative importance of vessels of low or high conductance.

The minimization of the function (9) is performed by finding the set of conductance $c_{e}$ which minimizes (9) for a given boundary condition $\boldsymbol{s}$. Following the ansatz in Ref. [18], we may formulate our minimization in the form of temporal adaptation rules for each vessel, where each element reacts to a local stimulus, instead of a single global optimization procedure. To derive a local adaption dynamic, we perform a gradient descent approach. This means we consider the temporal derivative $\Gamma$ as

$$
\frac{d \Gamma}{d t}=\sum_{j} \nabla_{c_{e}} \Gamma \cdot \partial_{t} c_{e}
$$

We want to ensure $\frac{d \Gamma}{d t} \leqslant 0$ and therefore that $\Gamma$ converges toward a local minimum. To do so, we may formulate the dynamical equations for $c_{e}$ as the negative gradient of $\Gamma$ :

$$
\partial_{t} c_{e} \propto-\nabla_{c_{e}} \Gamma=\left(\frac{f_{e}^{2}}{c_{e}^{2}}-a \gamma c_{e}^{\gamma-1}\right),
$$

where we used the definition of $\Gamma$ in Eq. (9). The dynamics in Eq. (11) allow for a continuous local adaptation of the vessel's state by consideration of its local flux, current conductance, and metabolic parameters $a, \gamma$. We extend this approach for interacting multilayer networks in a linear manner by adding up the metabolic cost of the individual systems involved and adding respective interaction terms. In our particular case, we do so for two flow networks with

$$
\Gamma=\Gamma_{12}+\Gamma_{1}+\Gamma_{2},
$$

where $\Gamma_{1}, \Gamma_{2}$ are given for each network by Eq. (9). The interaction term $\Gamma_{12}$ incorporates the geometrical nature of the system and encapsulates either a competition or symbiosis of the vessels of the two systems on a local basis as well. The exact nature of this interaction term as well as of the metabolic costs will be discussed in the next section in further detail, where we derive the dynamical systems in accordance with Eq. (11).

\section{Modeling intertwined vessel systems}

In this section, we connect the results of the previous sections to the relevant hydrodynamics and discuss the nature of the exact biological flows problem. The direct applicability of Eqs. (3) and (4) to biological systems becomes clear when considering the Hagen-Poiseuille law [46], which describes the volume flow rate $f$ of a fluid of viscosity $\eta$ at low Reynold numbers through a tube of length $l$ and radius $r$ as

$$
f=\left(\frac{\pi r^{4}}{8 \eta l}\right) \Delta p
$$

This provides us with a conductance $c=\frac{\pi r^{4}}{8 \eta l}$ by direct comparison with Ohm's law, with a fourth-order dependency of the radius $r$. We will here focus on radial adaptation and consider the special case of $l_{i}=$ const. for all vessels in either network. Hence, using Eqs. (9) and (13), we may rewrite the 

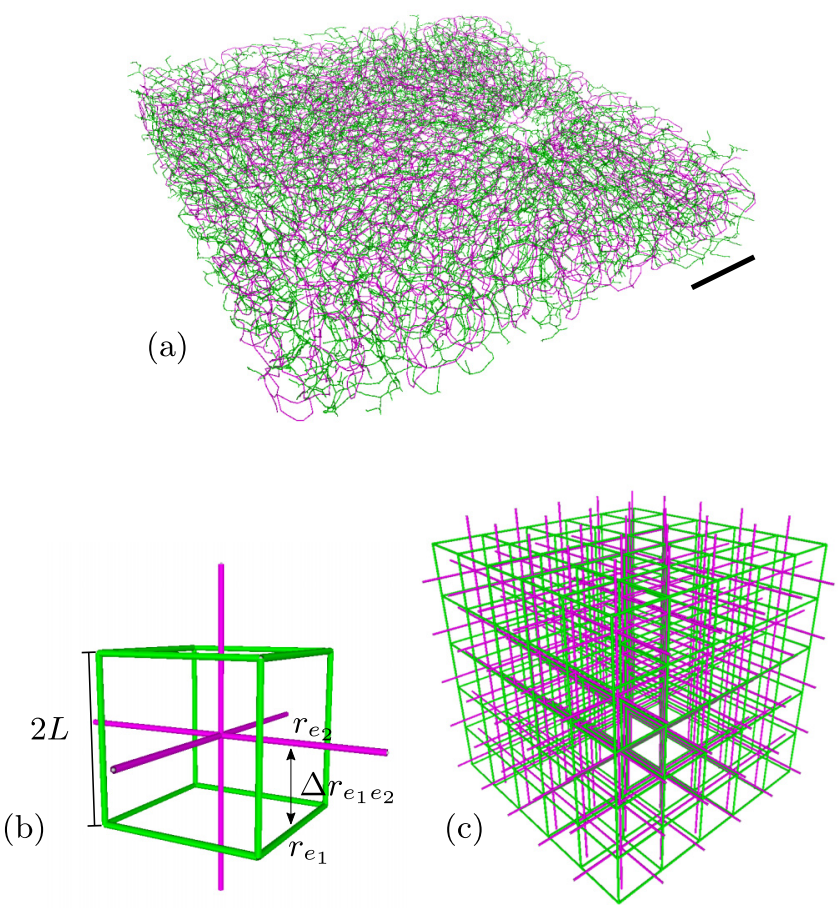

FIG. 1. Modeling biological intertwined structures: (a) Segment of bile canaliculi (green) and sinusoids (magenta) in the mouse's liver acinus (presented as network skeletons; see also Ref. [29]); scale bar is $200 \mu \mathrm{m}$. [(b), (c)] Model for intertwined, initial plexi: (b) (Cubic) unit cell and indication of spatial constraint for tube surfaces. (c) Complementary cubic lattices as a model for a space filling intertwined networks.

cost ansatz in (12) as

$$
\begin{aligned}
\Gamma= & \Gamma_{12}+\left(\frac{\pi}{8 \eta_{1} l_{1}}\right)\left[\sum_{e_{1}} r_{e_{1}}^{4} \Delta p_{e_{1}}^{2}+a_{1} r_{e_{1}}^{4 \gamma_{1}}\right] \\
& +\left(\frac{\pi}{8 \eta_{2} l_{2}}\right)\left[\sum_{e_{2}} r_{e_{2}}^{4} \Delta p_{e_{2}}^{2}+a_{2} r_{e_{2}}^{4 \gamma_{2}}\right] .
\end{aligned}
$$

From here on, we use the indices $i \in\{1,2\}$ for the two networks. Further, we only consider the specific case $\gamma_{i}=0.5$, which relates the metabolic cost directly toward volume for each vessel. Performing a minimization of the cost (14) at this point, without considering any interactions $\Gamma_{12}$, would result in each network to independently become dissipation minimized, constrained by its overall volume. We thus turn our attention to the interaction term $\Gamma_{12}$. In order to model interacting networks such as those found in the liver lobule [see Fig. 1(a)], we define a multilayer network consisting of two intertwined, yet spatially separate objects each consisting of edges $e_{i}$, with designated vessel radii $r_{e_{i}}$ see Figs. 1(b) and 1(c). Each edge in either network is affiliated with the set of closest adjacent edges of the other respective network. As all theses edges are simply tubes in our model, we define the distance between affiliated tube surfaces to be

$$
\Delta r_{e_{1} e_{2}}=L-\left(r_{e_{1}}+r_{e_{2}}\right),
$$

where $L$ is the initial distance of the abstract network skeletons (equal to distance in case of simultaneously vanishing radii). To model a system of blood vessels entangled with a secondary, secreting vessel network, we postulate that the respective tube surfaces must not fuse or touch directly, i.e., $\Delta r_{e_{1} e_{2}} \geqslant 0$. Subsequently, we construct the interaction term $\Gamma_{12}$ for the combined system as a power of the relative distance $\Delta r_{e_{1} e_{2}}$ :

$$
\Gamma_{12}=\frac{b}{2} \sum_{e_{1} e_{2}} F_{e_{1} e_{2}} \Delta r_{e_{1} e_{2}}^{\varepsilon}
$$

$$
\text { with } F_{e_{1} e_{2}}= \begin{cases}1 & \text { if edges } e_{1} \text { and } e_{2} \text { affiliated } \\ 0 & \text { else }\end{cases}
$$

with positive coefficient $b \geqslant 0$ and exponent $\varepsilon \in \mathbb{R}$ allowing us to switch between a repulsive or attractive behavior of the interaction (see Sec. II D), resembling either the competition for space or a mechanism to increase mutual contact. We have therefore arrived at the total cost function for the system:

$$
\begin{aligned}
\Gamma= & \frac{b}{2} \sum_{e_{1} e_{2}} F_{e_{1} e_{2}} \Delta r_{e_{1} e_{2}}^{\varepsilon} \\
& +\left(\frac{\pi}{8 \eta_{1} l_{1}}\right)\left[\sum_{e_{1}} r_{e_{1}}^{4} \Delta p_{e_{1}}^{2}+a_{1} r_{e_{1}}^{2}\right] \\
& +\left(\frac{\pi}{8 \eta_{2} l_{2}}\right)\left[\sum_{e_{2}} r_{e_{2}}^{4} \Delta p_{e_{2}}^{2}+a_{2} r_{e_{2}}^{2}\right],
\end{aligned}
$$

which we may now use to derive the dynamical system via gradient descent.

\section{Adaptation dynamics of intertwined vessel systems}

In this section, we discuss in detail the dynamical systems we intend to construct via the gradient descent approach on the basis of the cost function shown in Eq. (18). Calculating the gradient, we acquire the equations of motion for each network as

$$
\begin{aligned}
\partial_{t} r_{e_{1}} \propto & {\left[\Delta p_{e_{1}}^{2} r_{e_{1}}^{2}-\frac{a_{1}}{2}\right] r_{e_{1}} } \\
& +\frac{b \eta_{1} l_{1} \varepsilon}{\pi} \sum_{e_{2}} F_{e_{1} e_{2}} \Delta r_{e_{1} e_{2}}^{\varepsilon-1}, \\
\partial_{t} r_{e_{2}} \propto & {\left[\Delta p_{e_{2}}^{2} r_{e_{2}}^{2}-\frac{a_{2}}{2}\right] r_{e_{2}} } \\
& +\frac{b \eta_{2} l_{2} \varepsilon}{\pi} \sum_{e_{1}} F_{e_{1} e_{2}} \Delta r_{e_{1} e_{2}}^{\varepsilon-1} .
\end{aligned}
$$

The details of the derivation are given in Appendix A. It may be noted here that the terms $\Delta p_{i}^{2} r_{i}^{2}$ correspond to the wall shear stress exerted, manifesting itself in a positive growth feedback. The negative terms $\frac{a_{i}}{2}$ relate to the metabolic cost. This term imposes effectively a volume penalty on the system as growing vessels generate an increased negative feedback. The interaction term reacts to the relative vessel distance $\Delta r$ and imposes a feedback connected to the local neighborhood of each vessel which can be either positive (attractive coupling) or negative (repulsive coupling) depending on the choice of $\varepsilon$.

In order to perform a numerical evaluation of the resulting ordinary differential equation (ODE) system, we define a unit system and nondimensional parameters as follows: The 


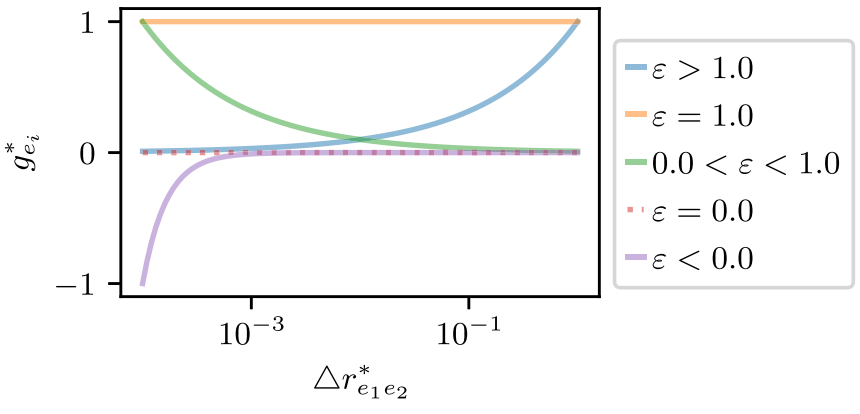

FIG. 2. Qualitative behavior of the coupling term $g_{e_{i}}^{*}$ as defined in Eqs. (21) and (22): The interaction poses a positive growth feedback for $g_{e_{i}}^{*}>0$ and a negative feedback for $g_{e_{i}}^{*}<0$. Function values of $g_{e_{i}}^{*}$ are presented here in dependence of the distance of a single arbitrary vessel pairing; curves are normalized for comparison.

radii and edge lengths in units of the grid distance $r_{e_{i}}=L r_{e_{i}}^{*}$, $l_{i}=L l_{i}^{*}$, the nodal in and outflow $s_{v_{i}}=\mu_{i} s_{v_{i}}^{*}$, the conductance $c_{e_{i}}=\eta_{i}^{-1} L^{3} c_{e_{i}}^{*}$ and hence pressure $\Delta p_{e_{i}}=\frac{\mu_{i} \eta_{i}}{L^{3}} \Delta p_{e_{i}}^{*}$, and the networks' edge surface distance $\Delta r_{e_{1} e_{2}^{\prime}}^{\varepsilon-1}=L^{\varepsilon-1} \Delta r_{e_{1} e_{2}}^{* \varepsilon-1}$. We define the timescale via the volume flow rates in the primal network as $t=\frac{L^{3}}{\mu_{1}} t^{*}$. Given positive proportionality constants $\chi_{i} \geqslant 0$ in the Eqs. (19) and (20), we define the effective temporal response parameters in either network as $\lambda_{0}^{i}=\frac{\chi_{1} L^{3}}{\mu_{1}}\left(\frac{\mu_{i} \eta_{i}}{L^{2}}\right)^{2}$. Further, we define the effective network couplings $\lambda_{1}^{i}=\frac{b|\varepsilon| L^{3+\varepsilon}}{\pi \eta_{i} \mu_{i}^{2}}$ and the effective volume penalties $\lambda_{2}^{i}=\frac{a_{i}}{2}\left(\frac{L^{2}}{\mu_{i} \eta_{i}}\right)^{2}$. We introduce an effective coupling $g_{e_{i}}^{*}$ term as

$$
\begin{aligned}
& g_{e_{1}}^{*}=\operatorname{sgn}(\varepsilon) l_{i}^{*} \sum_{e_{2}} F_{e_{1} e_{2}} \Delta r_{e_{1} e_{2}}^{* \varepsilon-1}, \\
& g_{e_{2}}^{*}=\operatorname{sgn}(\varepsilon) l_{i}^{*} \sum_{e_{1}} F_{e_{1} e_{2}} \Delta r_{e_{1} e_{2}}^{* \varepsilon-1},
\end{aligned}
$$

using the sign function $\operatorname{sgn}(\varepsilon)$. We thus arrive at the dimensionless form of the dynamical equations for each network:

$$
\partial_{t^{*}} r_{e_{i}}^{*}=\lambda_{0}^{i}\left(\Delta p_{e_{i}}^{* 2} r_{e_{i}}^{* 3}-\lambda_{2}^{i} r_{e_{i}}^{*}+\lambda_{1}^{i} g_{e_{i}}^{*}\right) .
$$

The coupling $g_{e_{i}}^{*}$ changes its qualitative behavior with variation of the exponent $\varepsilon$. We will consider the cases of an attractive coupling $\varepsilon>1$ and a repulsive coupling $\varepsilon<0$; see Fig. 2. We will further discuss the trivial case of an uncoupled system $\varepsilon=0$ (for which $\lambda_{1}=0$ ) for direct comparison with the $\mathrm{Hu}-\mathrm{Cai}$ model. Note that $\varepsilon=1$ will result in a constant $g_{e_{i}}^{*}$ corresponding to a positive constant background stimuli, as proposed in Ref. [21]. The case $0<\varepsilon<1$ results in a diverging attraction for nearby vessels, while it basically drops out for $\Delta r_{e_{1} e_{2}} \longrightarrow 1$. These numerically and heuristically unfavorable cases of $0<\varepsilon<1$ as well as the linear case $\varepsilon=1$ will be neglected hereafter.

It should be pointed out that this model ansatz could be used in the same manner to introduce distance dependent self-interactions for vessels of the same network. For example, we could construct a cost term $\Gamma_{i i}$ resulting in a ODE system similar to (23), displaying the same qualitative form of the coupling term $g_{e_{i}}^{*}$. Yet, a critical difference is the potential of congenial vessel membranes to merge upon contact. In real biological organisms, this actually presents an alternative vascular pruning mechanism in the endothelium [7] and subsequently leads to critical topology changes, which are beyond the scope of the investigation presented here.

\section{E. Incorporating flow fluctuations: Noisy, uncorrelated sink patterns}

Next, we assume the adaptation of the vascular networks depends on an averaged potential landscape instead of instantaneous configurations, which are bound to occur in real systems due to short-term metabolic changes or vessel blocking and/or damage. In other words, we assume a constant vessel radius between two adaptation events, while the flow rates change throughout the system due to changes in the sinks' magnitude, enabling us to substitute $\Delta p_{e}^{2} \rightarrow\left\langle\Delta p_{e}^{2}\right\rangle$ in Eq. (23). We thus implicitly assume a timescale separation between the radii adaptation (long-time changes, not to be confused with short-term contraction and/or dilation) and changes of hydrostatic pressure. We define fluctuations in accordance to the Corson model [14]. Subsequently, we will only consider $s$ configurations in which there exists one source node (here $v_{\text {source }}=0, s_{0}=-\sum_{v>0} s_{v}$ ) and all other nodes are randomly initialized sinks with the following characteristics:

$$
\begin{gathered}
\left\langle s_{v}\right\rangle=\mu_{v} \text { with } v>0 \\
\left\langle s_{v} s_{w}\right\rangle=\rho_{v w} \sigma_{v} \sigma_{w}+\mu_{v} \mu_{w} \text { with } v, w>0 .
\end{gathered}
$$

We assume the fluctuations are uncorrelated and follow the same probability distribution. We set for the mean $\mu_{v}=\mu$, standard deviation $\sigma_{w}=\sigma$, and correlation coefficient $\rho_{v w}=$ $\delta_{v w}$. We may subsequently calculate the average squared pressure:

$$
\left\langle\Delta p_{e}^{2}\right\rangle=\mu^{2} \phi_{e}+\sigma^{2} \delta \phi_{e},
$$

where the function $\phi_{e}$ describes the squared pressure in the case of a constant source-sink landscape in the absence of any variance $\sigma^{2}$. Further, the function $\delta \phi_{e}$ describes the pressure perturbation caused by fluctuations with variation $\sigma^{2}$ in analogy to the Hu-Cai model's heuristic fluctuation ansatz [18]. For the full derivation of Eq. (26) and the detailed computation of $\phi_{e}, \delta \phi_{e}$, see Appendix B. Using this approach, we prevent shunting and the generation of spanning trees, which is caused by the typical "single source and multiple sinks" setting. Further, using this ansatz one also prevents accidentally partitioning the graph, which can happen when realizing the sink-source configurations one by one [3].

We find this ansatz in particular fitting to model the liver lobule system, as sinusoids are fenestrated structures (meaning the vessel wall is perforated). Additionally, bile and water is frequently secreted by hepatocytes (cells forming the bulk of the tissue and the basic metabolic unit in the liver) into bile canaliculi. On the other hand, one may argue that the fluid leak in the sinusoidal system is negligible in comparison to the overall throughput rate, and an additional distinguished sink would have to be placed at the opposing end of the plexus, extracting the majority of fluid. Here we neglect this factor as one major sink would merely generate one (or a small number of) distinguished large vessel(s), without any further impact on the topological complexity of the rest of the networks. 
We incorporate these flow fluctuations with an effective fluctuation strength $\lambda_{3}^{i}=\frac{\sigma_{i}^{2}}{\mu_{i}^{2}}$ in Eq. (23):

$$
\partial_{t^{*}} r_{e_{i}}^{*}=\lambda_{0}^{i}\left(\left(\phi_{e_{i}}^{*}+\lambda_{3}^{i} \delta \phi_{e_{i}}^{*}\right) r_{e_{i}}^{* 3}-\lambda_{2}^{i} r_{e_{i}}^{*}+\lambda_{1} g_{e_{i}}^{*}\right)
$$

Thereby $\lambda_{3}^{i}$ scales the strength of pressure perturbations, which effectively impose an increase in the wall shear stress term in Eq. (27).

\section{F. Order parameters for network remodeling}

In order to quantify the topological changes occurring in an adapting system, we monitor the relative cycle density as an indicator of redundancy. Loosely, one may identify the number of cycles in a network in the following simple way: If we assume that the network's representing simple graph is one connected component of $n$ vertices and $m$ edges, then we only need $n-1$ edges to connect every vertex into a spanning tree without a single cycle, while each additional edge added from here on will form a cycle. Thus, the total amount of such cycles in a network $z$ is the number of excess edges from the total number of edges:

$$
z=m-(n-1) .
$$

Strictly speaking, $z$ is the amount of independent cycles (also referred to as nullity) and may be calculated for any simple multicomponent graph [47]. We use this metric in the following way: We solve the dynamical systems (27) until the networks reach a stationary state. The initial graph structure this process starts from is called a plexus and represents in biological terms the rudimentary vessel network which is formed before perfusion sets in. During this optimization, we mark edges whose radius falls below a critical threshold $r_{c}^{*}$. These edges are no longer updated and are considered to have a radius of virtually zero (though for computational reasons they are here set to $r_{c}^{*}=10^{-21}$ ). We call such edges "pruned" which corresponds to the biological phenomenon of having a vessel degenerate and collapse. Then we remove all pruned edges and disconnected vertices from the networks and calculate the remaining number of cycles according to Eq. (28). We then define the relative nullity of an equilibrated network,

$$
\varrho=\frac{m-n+1}{z_{0}}
$$

as an order parameter, where $z_{0}$ is the initial number of independent loops before adaptation. Hence $\varrho=0$ corresponds to a treelike network while $\varrho>0$ captures the relative amount of redundancy in comparison to the initial plexus.

\section{NUMERICAL EVALUATION OF THE MODEL FRAMEWORK}

In this section, we present the simulation results acquired by solving the dynamical system (27) numerically until the system reaches a stationary state. Of particular interest is the final network's topology, i.e., its relative reticulation characterized by the order parameter $\varrho$. We study in detail the dependence of $\varrho$ on the coupling $\lambda_{1}$, the volume penalty $\lambda_{2}$, and the fluctuation rate $\lambda_{3}$. Primary focus is on the interplay of coupling and fluctuation and how the underlying threedimensional lattice topology affects the remodeling process. All diagrams shown represent the results for one of the two intertwined networks; the results are symmetric for the other network due to a symmetric choice of the effective parameters $\lambda_{1}^{i}, \lambda_{2}^{i}$, and $\lambda_{3}^{i}$; see Appendix $\mathrm{C}$ for further details.

As underlying graph topology for the initial state networks, we take the graph skeletons of the triply periodic minimal surfaces P ["dual" simple cubic; see Fig. 1(c)], D ("dual" diamond cubic), and G ("dual" Laves) [48]. These systems present highly symmetric and complementary space-filling graphs and enable us to construct well-defined intertwined networks with clear local edge affiliations. The initial edge radii are chosen randomly. We set the fluctuation rates identical $\lambda_{3}^{1}=\lambda_{3}^{2}$ as well as the coupling strength $\lambda_{1}^{1}=\lambda_{1}^{2}$. We did not find any qualitative differences in our results among different intertwined topologies. We thus present here the results for the simplest starting topology, the intertwined cubic lattices.

\section{A. Fluctuation-induced nullity transitions independent of volume penalty and topology}

First, we test the original Hu-Cai model in combination with Corson's fluctuation approach, i.e., the uncoupled case of $\varepsilon=0$ for three-dimensional lattices. We do so for two reasons: first, to confirm the robustness of the adaptation mechanic for a plexus represented by nonplanar graphs in a manner similar to that in Ref. [20], and second, to confirm the independence of the fluctuation-induced nullity transition from the volume penalty $\lambda_{2}$, as indicated in Ref. [18]. To do so, we calculate the adaptation with a single corner source node (sinks otherwise) for a systematic scan of $\lambda_{2}$ and $\lambda_{3}$ (see Fig. 3).

Indeed, we recover the transition from tree-like configurations for $\lambda_{3} \leqslant 1$ [Fig. 3(c)] toward states exhibiting fluctuation-induced loops for large $\lambda_{3}$ [Fig. 3(d)]. The emerging transition is of logarithmic nature, effectively saturating for $\lambda_{3} \approx 10^{4}$. In particular, we confirm that only an increase in the fluctuation ratio, $\lambda_{3}$, results in an increase in the nullity [Fig. 3(a)]. The continuous transition observed is independent of the system's effective volume penalty $\lambda_{2}$ [Fig. 3(b)]. Note, however, that the parameter $\lambda_{2}$ influence the final vessel diameter as well as the timescales for reaching the stationary state (as does the system size if the number of identical sinks scales with system size). Further, we confirm that the adaptation mechanism reproduces the qualitative network topologies in three-dimensional lattices as found before in planar graphs $[14,18]$. From here, we turn our attention to the fluctuationinduced transition in comparison to the new spatial coupling.

\section{B. Repulsive coupling shifts and rescales fluctuation-induced nullity transition}

To consider the novel, spatially coupled cases, we systematically scan the effective network coupling and flowfluctuation parameters. In this section, we will focus on the case of repulsive interactions, i.e., setting the coupling exponent to $\varepsilon=-1$. Of particular interest is the influence of the 

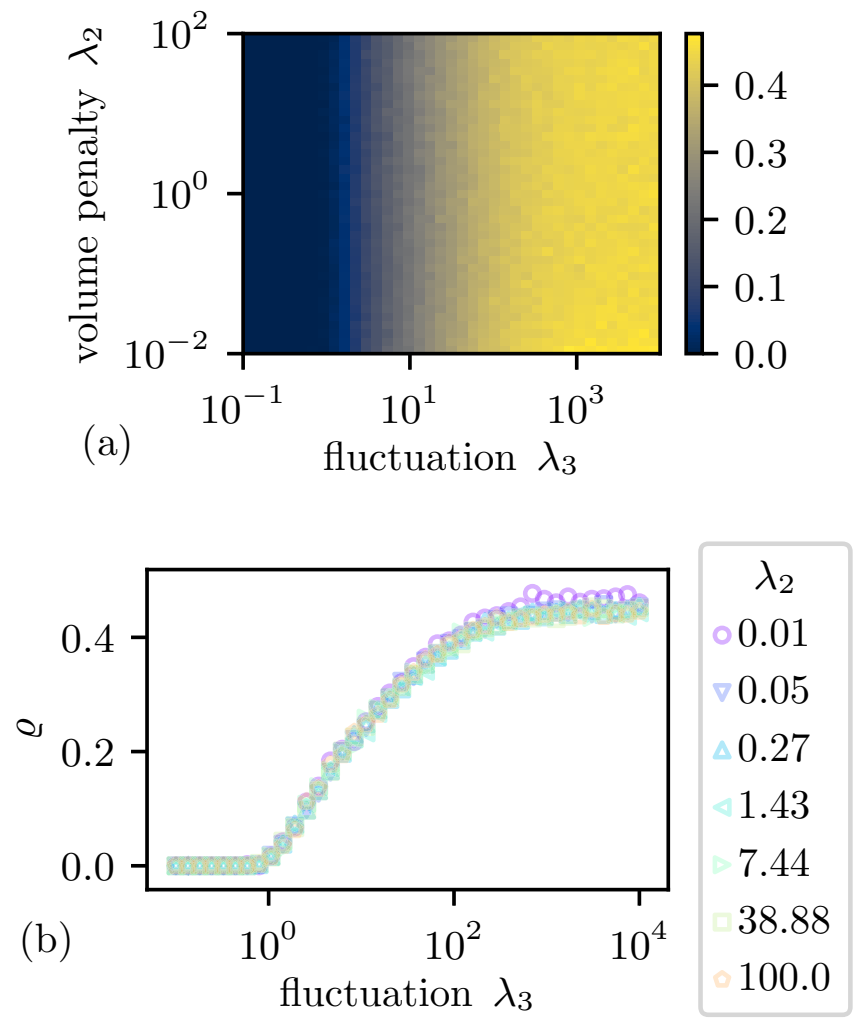

(c)
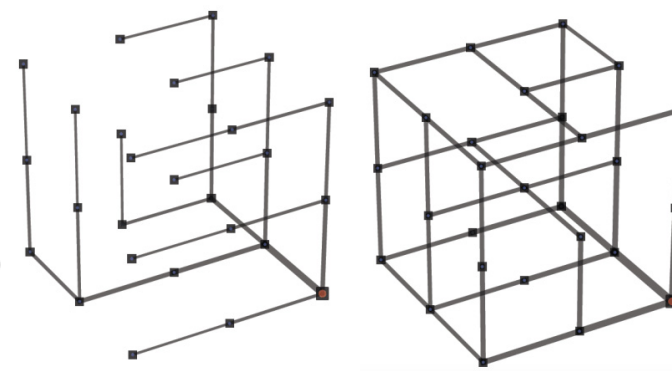

FIG. 3. Nullity $\varrho$ state diagram and transition trajectories indicate fluctuation $\lambda_{3}$ induced nullity transition to be independent of volume penalty $\lambda_{2}$ : [(a), (b)] Uncoupled adapting networks display continuous, logarithmic $\lambda_{2}$-independent nullity $\varrho$ transitions in uncoupled system. Further shown are the stationary network configurations for $\lambda_{2}=1$ with (c) $\lambda_{3}=10^{-1}$ and (d) $\lambda_{3}=10^{2}$. The edge thickness is representative for the relative tube radius $\rho$. Sinks are marked as blue dots, with the source as a large red circle.

negative feedback this interaction introduces to the dynamical system (27).

First, we see the fluctuation-induced nullity transition (as observed for the uncoupled system) to be preserved for weak couplings, $\lambda_{1}<10^{4}$. The full state diagram [Fig. 4(a)] shows that the system's nullity may be influenced above that threshold not only by the rate of fluctuations $\lambda_{3}$ but also by the mutual repulsion of the two networks. Figure 4(b) shows the corresponding $\lambda_{1}$-induced breakdown, displaying the possibility of nullifying any reticulated structures by increasing the repulsive coupling strength in the system. Nevertheless, it seems that the influence of the repulsion is weaker in comparison, needing coupling parameters to be orders of magnitudes larger than the fluctuation rates. On the other hand, we also find the fluctuation-induced nullity onset to be continuous, as it was for the uncoupled system. Starting from a treelike state at small fluctuations and increasing monotonically in a logarithmic manner beyond a critical $\lambda_{3} \geqslant \lambda_{c}$ [Fig. 4(c)], we have the $\varrho$ trajectory eventually saturating for large fluctuation rates $\lambda_{3}>10^{3}$ toward a maximal nullity $\varrho_{\max }$. This leaves the network in a reticulated state, still displaying a visible vessel hierarchy toward the source. We can recover almost treelike network states for increased repulsion rates $\lambda_{1}$, even losing the typical vessel hierarchy toward the source; see Fig. 5. This increase in $\lambda_{1}$ further shifts the onset of nullity. To quantify these shifts, we acquire the critical $\lambda_{c}$ by identifying the departure from zero in Fig. 4(c). The critical point $\lambda_{c}$ seems to monotonically increase with the coupling parameter $\lambda_{1}$. Following up on this observation, we extrapolated the onset of saturation in Fig. 4(c) by means of sigmoidal fits. The shifts of these indicators are shown in Fig. 4(d), displaying a general increase of both the critical value and the saturation for increasing $\lambda_{1}$. Using the acquired critical values $\lambda_{c}$, we rescale the trajectories of Fig. 4(c) between the onset of the nullity transition and its saturation, as shown in Fig. 4(e). Introducing the reduced fluctuation parameter $\frac{\lambda_{3}-\lambda_{c}}{\lambda_{c}}$, we find the trajectories to collapse on a single master curve, following a trivial logarithmic law as

$$
\varrho\left(\lambda_{1}, \lambda_{3}\right) \approx \kappa\left[\lambda_{1}\right)\left(\log _{10}\left(\frac{\lambda_{3}-\lambda_{c}}{\lambda_{c}}\right)-1\right]
$$

with the coupling-dependent scaling factor $\kappa\left(\lambda_{1}\right)$ acquired by interpolation of the data by Eq. (30). We find $\kappa$ to be a decreasing function the coupling $\lambda_{1}$ as shown in Fig. 4(f). This shows that the nullity breakdown and shift can be tuned for any given fluctuation rate by the coupling alone. Further, the negative feedback (caused by the repulsion of the two networks) does not cause any shunting (i.e., collapse and disconnection of large sections of the networks) whatsoever.

For all simulations shown, we set the response $\lambda_{0}^{1}=$ $\lambda_{0}^{2}=10^{-4}$ and volume penalty $\lambda_{2}^{1}=\lambda_{2}^{2}=10^{6}$ (providing reasonable computation times for reaching stationary states and preventing the problem from becoming too stiff). The initial edge radii are chosen randomly and are subsequently continuously monitored to fulfill

$$
0<r_{e}^{*}+r_{e^{\prime}}^{*}<1
$$

in order to prevent negative radii or radii combinations corresponding to intersections.

\section{Attractive coupling induces a nullity transition, fully recovering initial plexus}

We now consider intertwined networks with an attractive spatial coupling. We initialize the system with a positive coupling exponent, $\varepsilon=3$. We are particularly interested how the positive feedback this interaction introduces to the dynamical system (27) interacts and compares with the fluctuationinduced nullity transition.

In Fig. 6, we show the resulting nullity state diagrams and transition curves for a systematic scan of the couplings and fluctuation rates. For increasing coupling strength $\lambda_{1}$, we see the emergence of a new nullity transition for $\lambda_{1}>\lambda_{c}$, as 

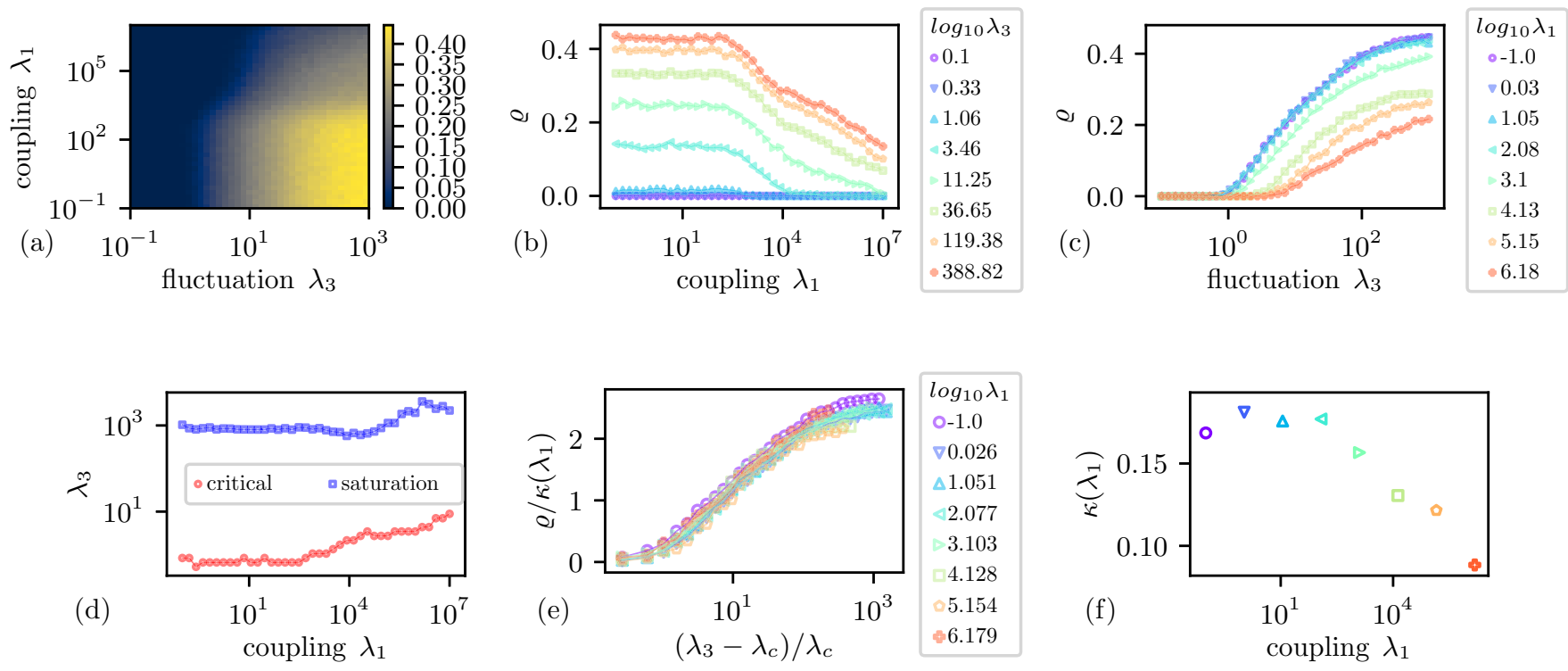

FIG. 4. Nullity $\varrho$ state diagram and transitions shown for repulsively coupled networks $(\varepsilon=-1)$ display a nullity breakdown and rescaling of the transition trajectories: (a) Coupling vs fluctuation, $\left(\lambda_{1}, \lambda_{3}\right)$ diagram with nullity $\varrho$ color map. (b) Coupling $\lambda_{1}$ induced nullity breakdown for varying fluctuation $\lambda_{3}$. (c) Fluctuation $\lambda_{3}$ induced nullity onset for varying coupling $\lambda_{1}$ (d) Critical $\lambda_{3}$ for onset of nullity transition for $\lambda_{1}$ variation and extrapolated saturation of nullity transition. (e) Single curve collapse near fluctuation-induced nullity onset $\lambda_{c}$ and scaled nullity $\varrho / \kappa\left(\lambda_{3}\right)$. We find the transition to be approximated by $\varrho\left(\lambda_{1}, \lambda_{3}\right) \approx \kappa\left(\lambda_{1}\right)\left(\log _{10}\left(\frac{\lambda_{3}-\lambda_{c}}{\lambda_{c}}\right)-1\right)$. (f) Coupling-dependent scaling factor $\kappa\left(\lambda_{1}\right)$, as derived from linear interpolation of rescaled transition curves.

visualized, for example, in Fig. 7. This transition recovers virtually the entire initial plexus for $\lambda_{1} \rightarrow 10^{4}$ [Fig. 6(b)]. Furthermore, this transition is significantly sharper than the fluctuation-induced case as it does not occur on a logarithmic scale. Any increase in fluctuations, $\lambda_{3}$, generates positive offset of the nullity curve [Fig. 6(b)], indicating a constructive superposition of the mechanisms at hand. Yet, the trajectory's general form seems well preserved, while the saturation level is reduced for increased $\lambda_{3}$. Once again, we determine the transition's onset and saturation regime; see Fig. 6(d). To do so for the onset, we calculate the trajectories' root of the onset after subtracting the trajectories' offset. The saturation regime is extrapolated via a sigmoidal fit. As in the previous section. we are able collapse the trajectories onto a single master curve (a)

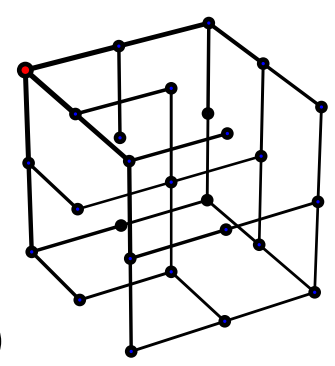

(b)

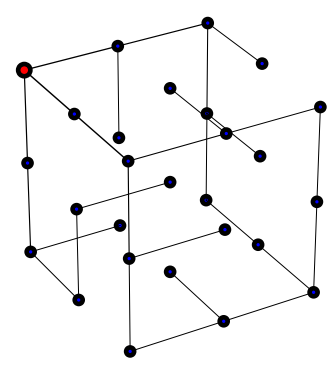

FIG. 5. Increased repulsive coupling $\lambda_{1}(\varepsilon=-1)$ causes nullity breakdown and overall reduction in edge thickness, shown here the transition for (a) $\lambda_{1}=10^{2}$ and (b) $\lambda_{1}=10^{8}$. Stationary network configurations shown for $\lambda_{2}=10^{6}$ and $\lambda_{3}=10$. The edge thickness is representative of the relative tube radius $r^{*}$. Sinks are marked as blue dots, with the source as a large red circle.
[Figure 6(e)] by following a trivial linear law:

$$
\varrho\left(\lambda_{1}, \lambda_{3}\right) \approx \kappa\left(\lambda_{3}\right)\left(\frac{\lambda_{1}-\lambda_{c}}{\lambda_{c}}\right)+\varrho_{0}\left(\lambda_{3}\right)
$$

with rescaled $x$ axis $\frac{\lambda_{1}-\lambda_{c}}{\lambda_{c}}$. The $\lambda_{3}$ dependency of the respective scaling parameters $\kappa\left(\lambda_{3}\right), \varrho_{0}\left(\lambda_{3}\right)$ are shown in Fig. 6(f). We note here that these curves are slightly different from the complementary network in the case of cubic lattice topology as they are considerably more spread out; see Appendix C for details. This phenomenon does seem to be topology dependent, as it is not present in the cases of Lave graphs or diamond lattices. The fluctuation-induced nullity transition, on the other hand, is in some sense perturbed beyond the $\lambda_{1}$ transition; see Fig. 6(c). It seems an underlying competition of mechanisms is observable for large $\lambda_{3}$ as the level of saturation is reduced, as can be directly seen in the scale factor $\kappa$; see Fig. 6(f). Nevertheless, this interplay between different positive feedback mechanisms creates multiple nullity states [see Fig. 6(a)], tuning the structures between spanning trees and partially reticulated and fully recovered plexus.

For all simulations, we set the response $\lambda_{0}^{i}=10^{-4}$ and volume penalty $\lambda_{2}^{i}=10^{6}$ as in the previous section. The parameter $\lambda_{3}$ is only considered here for $\lambda_{3}<300$ as affiliated edge pairs will violate the contact condition (31) beyond this range.

\section{GENERALIZING MURRAY'S LAW}

Our model of spatial coupling also points to a form of geometric law at vessel branchings. Recall Murray's law, which connects the radii $r_{0}$ of a parent vessel splitting into at least 

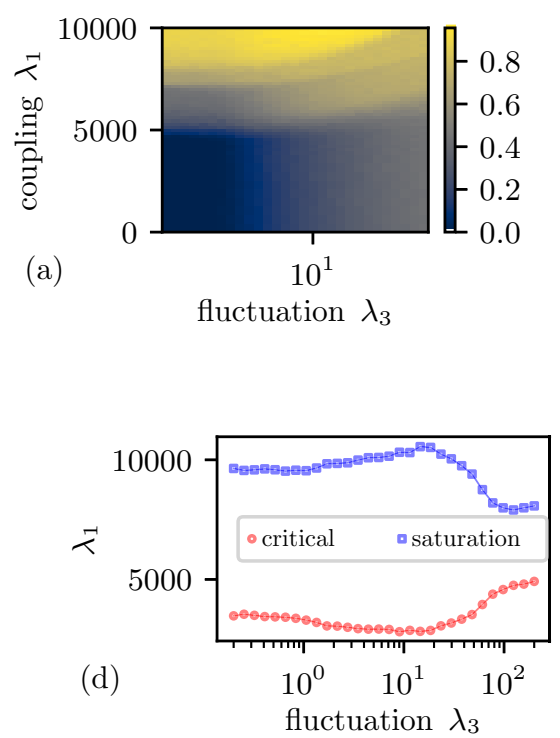
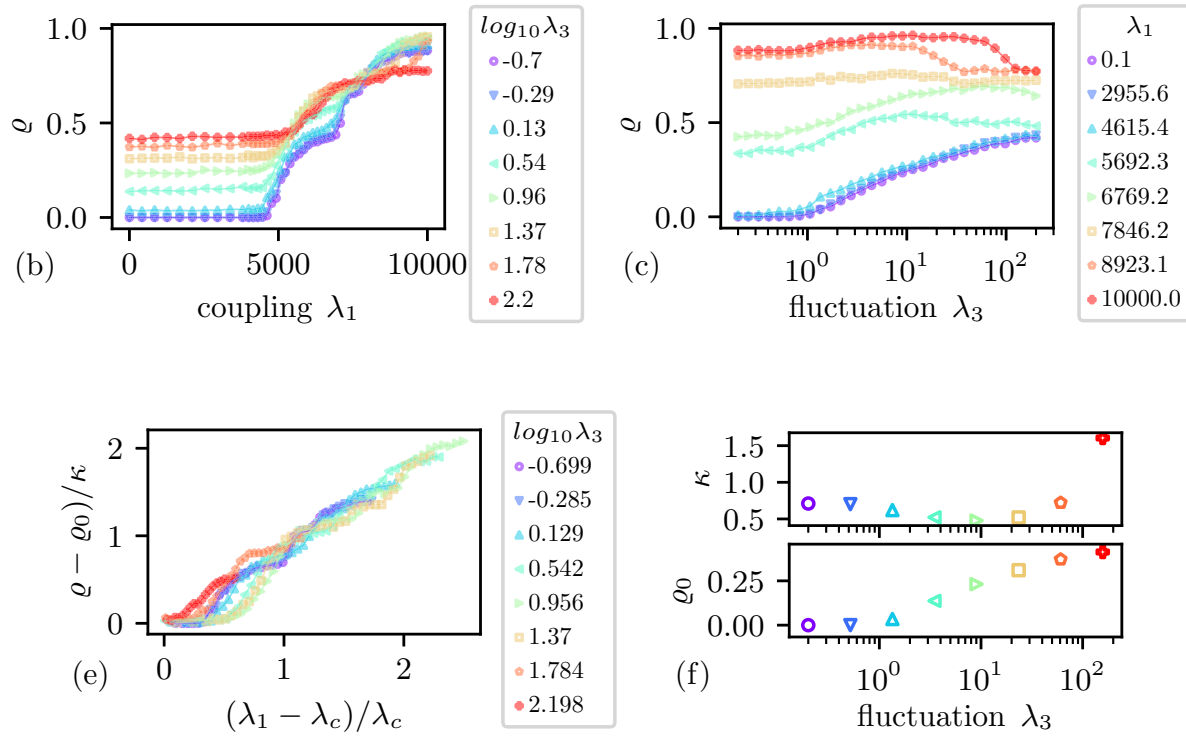

FIG. 6. Nullity $\varrho$ state diagram and transitions shown for attractively coupled networks $(\varepsilon=3)$ display a new topological transition: (a) Coupling vs fluctuation, $\left(\lambda_{1}, \lambda_{3}\right)$ diagram for nullity $\varrho$ color map. (b) Coupling $\lambda_{1}$ induced nullity transition for varying fluctuation $\lambda_{3}$. (c) Fluctuation $\lambda_{3}$ induced nullity transition for varying coupling $\lambda_{1}$. (d) Critical $\lambda_{1}$ for onset of nullity transition for $\lambda_{3}$ variation and extrapolated saturation of nullity transition. (e) Single curve collapse near coupling-induced nullity onset $\lambda_{c}$ and scaled nullity $\left[\varrho-\varrho_{0}\left(\lambda_{3}\right)\right] / \kappa\left(\lambda_{3}\right)$. We find the transition to be approximated by $\varrho\left(\lambda_{1}, \lambda_{3}\right) \approx \kappa\left(\lambda_{3}\right)\left(\frac{\lambda_{1}-\lambda_{c}}{\lambda_{c}}\right)+\varrho_{0}\left(\lambda_{3}\right)$. (f) Coupling-dependent scaling factor and fluctuation-induced nullity offset $\kappa\left(\lambda_{3}\right), \varrho_{0}\left(\lambda_{3}\right)$, as derived from linear interpolation of rescaled curves.

two child branches with radii $r_{1}, r_{2}$ as

$$
r_{0}^{\alpha}=r_{1}^{\alpha}+r_{2}^{\alpha} \text {. }
$$

In the original formulation, an exponent of $\alpha=3$ was predicted as the outcome of a cost optimization process [10], which relates directly to the dissipation-volume minimization procedure discussed in Sec. II B. Further, rescaled cost models [12] which consider cost variations via an exponent $c_{e} \rightarrow c_{e}^{\gamma}$ suggest

$$
\alpha=2(\gamma+1)
$$

while discarding flow fluctuations. We illustrate here the problems one encounters when testing these power laws for real intertwined structures such as sinusoids and bile canaliculi in the mammalian liver. Further, we introduce a generalized form (a)

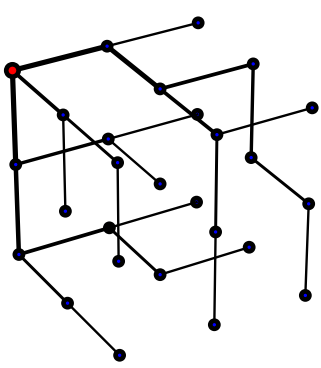

(b)

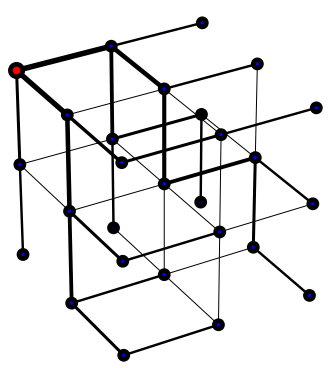

FIG. 7. Increased attractive coupling $(\varepsilon=3)$ creates an additional, local positive feedback which fixes overall nullity to the point of total plexus recover; shown here is the transition for (a) $\lambda_{1}=1$ and (b) $\lambda_{1}=10^{4}$. Stationary network configurations shown for $\lambda_{2}=10^{6}$ and $\lambda_{3}=10^{-1}$. The edge thickness is representative for the relative tube radius $r^{*}$. Sinks are marked as blue dots, with the source as a large red circle. of Murray's law which takes into account fluctuations as well as geometric coupling. We then use this form to estimate the interaction parameters of the real system heretofore inaccessible to experimental investigation.

The data sets were acquired from collaborators at the MPICBG in the following way: Mouse livers from adult mice were fixed by transcardial perfusion, sectioned into $100-\mathrm{mm}$ serial slices, optically cleared, and immunostained, as described in Ref. [29]. To visualize the different tissue components, the tissue sections were stained for nuclei (DAPI), cell borders (Phalloidin), bile canaliculi network (CD13), and the extracellular matrix (ECM, fibronectin and laminin) facing the sinusoidal network [49]. High-resolution images of the liver lobule (central vein, portal vein axis) were acquired by using confocal microscopy with a $63 \times / 1.3$ objective $(0.3-\mu \mathrm{m}$ voxel size). Finally, the resulting images were segmented and network skeletons calculated with the Motion Tracking software as described in Refs. [29] and [50].

\section{A. Classical Murray's law inadequate for reticulated, intertwined vessel structures of the liver lobule}

We test Murray's law for the network skeletons of the sinusoids and bile canaliculi in the liver lobule by fitting the exponent $\alpha$ in Eq. (33) for every branching of degree 3 (Y branching). There is significant deviation from the predicted exponent $\alpha=3$ (Fig. 8). The modes of the acquired, lognormally distributed fit exponents are $\alpha \approx 3.75$ for sinusoids and $\alpha \approx 3.33$ for bile canaliculi. As capillary systems were already known to defy the cubic relationship [25], we suspect this mismatch to be correlated with the reticulated nature of these network types. Further, one expects other mechanisms than mere dissipation-volume minimization will be involved, making these deviations not well described by the cost 

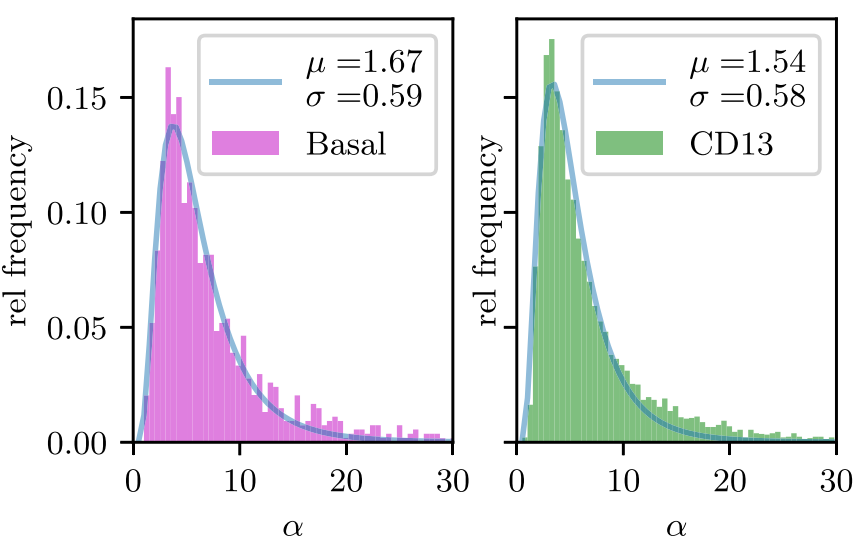

FIG. 8. Experimental falsification of Murray's law for capillary vessels in liver lobuli: Exponent distributions found for (33) and respective log-normal fits. The distributions modes are used to estimate the exponents $\alpha$. Sinusoidal systems (basal marker) with mode $\alpha=e^{\mu-\sigma^{2}} \approx 3.75$ and bile canaliculi systems (cd13 marker) with mode $\alpha=e^{\mu-\sigma^{2}} \approx 3.33$.

exponent $\gamma$ alone. Indeed, in accordance with Eq. (34), one would deduce $\gamma<1$ for the given liver lobule data sets, which is in direct contradiction with the rescaled cost model [12], which predicts an exponent $\gamma$-induced nullity transition only for $\gamma \geqslant 1$. On the other hand, this could potentially be circumvented if fluctuation-induced loops are considered as well $[13,14]$. However, to our knowledge it has not yet been discussed how such fluctuations alter Murray's law.

We deduce from our pruning model a set of coefficients, $a_{e}$, which are dependent on their corresponding edge's neighborhood and the respective coupling strength, as well as the global structure of sinks and sources (which were assumed to be uncorrelated and identically distributed). This procedure greatly alters the form of Eq. (33) and we derive a geometric law from the steady states of the ODEs in (27). We recover the cubic exponent of the original model with coefficient corrections that depend on the strength of fluctuations and spatial coupling:

$$
\begin{aligned}
\sum_{e} a_{e} r_{e}^{*, 3} & =1 \\
\text { with } a_{e} & = \pm \sqrt{\frac{\lambda_{2}-\lambda_{1} r_{e}^{*,-1} g_{e}^{*}}{1+\lambda_{3} \frac{\delta \phi_{e}^{*}}{\phi_{e}^{*}}}} .
\end{aligned}
$$

The effective coupling $g_{e}^{*}=\operatorname{sgn}(\varepsilon) \sum_{e^{\prime}} F_{e e^{\prime}} \Delta r_{e e^{\prime}}^{* \varepsilon-1}$, and squared edge potentials $\phi_{e}^{*}, \delta \phi_{e}^{*}$ are defined as in Sec. II. This law (35) may be further generalized in case of more complicated flow landscapes; see Appendix D.

\section{B. High-accuracy prediction of interaction parameters for ideal Kirchhoff networks}

We tested the feasibility of our generalized Murray's law (35) by simulating the pruning on a dual Laves graph topology (3-regular), with $n=206\left(n^{\prime}=210\right)$ vertices and $m=259$ $\left(m^{\prime}=261\right)$ edges, and setting the parameters symmetrically to $\lambda_{1}^{i}=10^{4}, \lambda_{2}^{i}=10^{6}, \lambda_{3}^{i}=10^{2}$. The sources were positioned in random vertices of the system. Edges of the respective networks were affiliated with each other by finding the nearest neighbors of edges inside a perimeter $\delta$. We numerically [51] find the roots of Eq. (35) for a set of positive definite $\lambda_{1}^{i}, \lambda_{2}^{i}, \lambda_{3}^{i}$. As we do not intend to use information on the direction of the currents at the sink nodes (as this information is not available in the real system), we solve Eq. (35) for the seven relevant sign permutations at each branching; see Appendix D, Fig. 14. For further evaluation, only the fit of highest quality (function value) is used. We use a logarithmic rescaling in order to find a symmetric representation of the histogram's data and doing so we fit a normal distribution $\mathcal{N}(\mu, \sigma)$ to the histogram's maxima. We use the terms $\mu_{i}\left(\log _{10} \lambda_{i}\right) \pm \sigma_{i}\left(\log _{10} \lambda_{i}\right)=\mu_{i} \pm \sigma_{i}$ to denote the characteristics of the distributions of $\log _{10} \lambda_{i}$. We find strong agreement with the actual parameters for both networks $\mu_{1} \pm \sigma_{1}=4.0 \pm 0.004, \mu_{2} \pm \sigma_{2}=6.0 \pm 0.007$, and $\mu_{3} \pm \sigma_{3}=1.999 \pm 0.003$; see Fig. 9(a) (depicted here only for the case of repulsive coupling $\varepsilon=-1$ ). We also tested for an abundance of multiple sources distributed throughout the system (acting as identical copies of each other). We find our procedure recovers the relevant model parameters in these cases as well [Fig. 9(b)]. We found in the same way good agreement for the case of attractive coupling with $\mu_{1} \pm \sigma_{1}=4.0 \pm 0.012, \mu_{2} \pm \sigma_{2}=5.999 \pm 0.01$, and $\mu_{3} \pm \sigma_{3}=1.998 \pm 0.008$.

\section{Limited estimation of interaction parameters for sinusoidal networks}

Finally, we use the extracted graph skeletons of the sinusoids and bile canaliculi in the vicinity of a central vein in the mouse liver to test the generalized Murray's law on a real intertwined vessel system. The vascular structure is represented by sets of vertices and edges bearing the positional and radial information of the respective vasculature. We use the same approach as in the prior section to estimate the coupling $\lambda_{1}$, volume penalty $\lambda_{2}$, and fluctuation rate $\lambda_{3}$ for the sinusoidal system. We stress that no information about the actual flow nor the exact point of flow injection or drainage was available. Hence, we first make some simplifying assumptions about the positions of sinks and sources as well as cropping the network skeletons to reduce their complexity: First, we identify the vertices in the sinusoidal network which are closest to the central vein and label them as sinks. The geometric center of mass of these vertices is calculated and used as the center of a sphere of radius $R$, representing the range of interest. Any other components, vertices, or edges of any network positioned outside this perimeter are discarded (here $R=390 \mu \mathrm{m}$, in order to keep the resulting graphs at a moderate size for computational purposes). Next, all branching points in the sinusoidal network are identified, as are all paths $\boldsymbol{p}=\left(e_{i}, \ldots, e_{j}\right)$ consisting of edges $e_{i}$ which start from these points. We proceed for the canaliculi the same way and check for each segment of a path whether there is another segment of another network's path inside a perimeter $\delta$ (here chosen as $30 \mu \mathrm{m}$ ). If so, these paths count as affiliated. We merge all edges along a path into a single edge by using the conventional addition theorems for series of resistors in Kirchhoff networks. Proceeding like this, we end up with a reduced sinusoidal network, with $n=318$ vertices and 

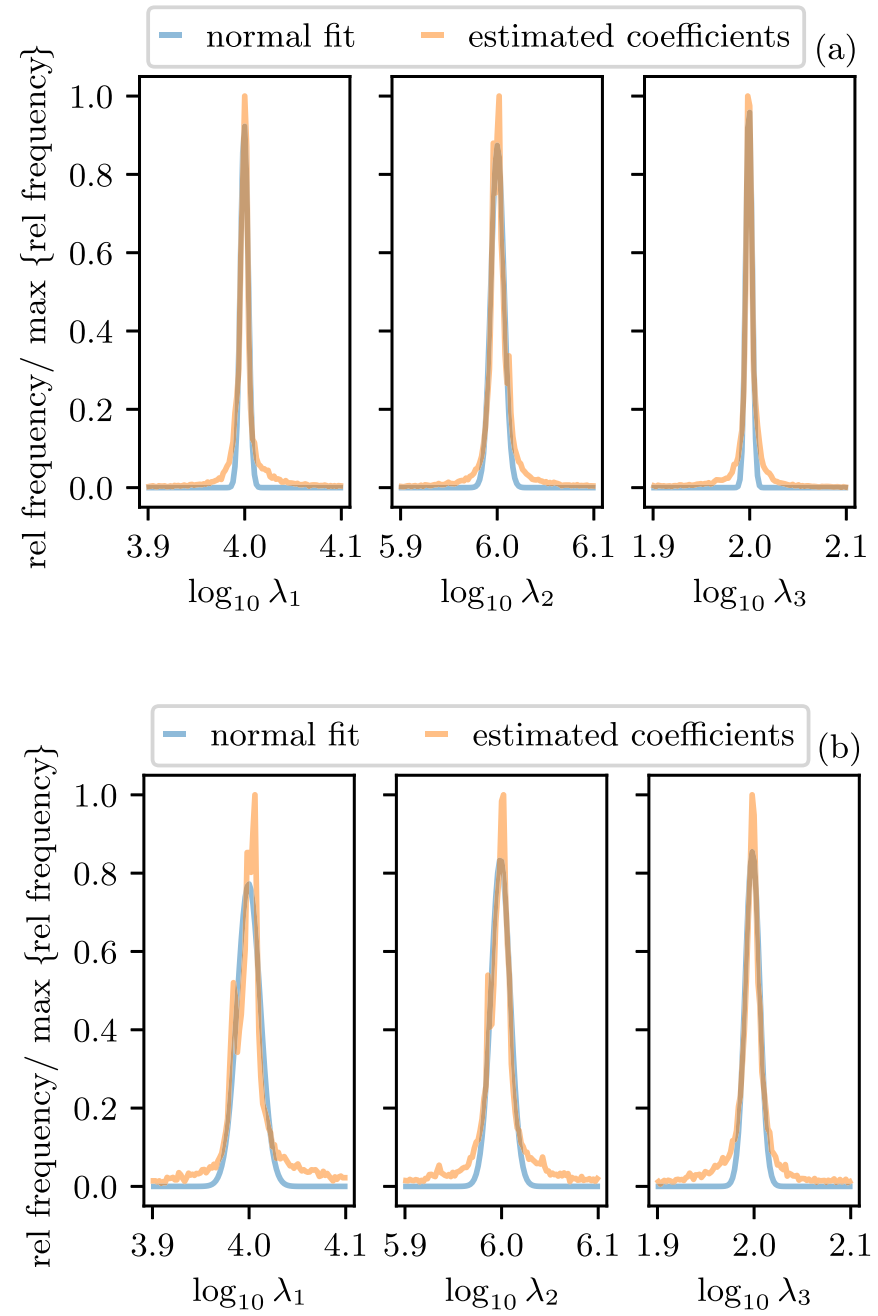

FIG. 9. High-accuracy extraction of interaction parameters is feasible for ideal Kirchhoff networks based on Eq. (35): Shown here is a test of coupled ideal Kirchhoff networks, the system was initialized symmetrically with coupling $\lambda_{1}=10^{4}$, volume penalty $\lambda_{2}=10^{6}$, and fluctuation $\lambda_{3}=10^{2}$ for the repulsive case $\varepsilon=-1$ and different sink-source landscapes. Histograms and fits $\mathcal{N}(\mu, \sigma)$ presenting the estimated simulation parameters $\lambda_{i}$. (a) Single-source and multisink system. (b) Multisource and multisink system.

$m=452$ edges. For further details on the reduction procedure, see Fig. 15 in Appendix D.

We find the parameters $\lambda_{1}, \lambda_{2}, \lambda_{3}$ numerically by solving Eq. (35) for randomly sampled tuples of branching points. We do so for a range of exponents $\varepsilon$, under consideration of all sign permutations for $a_{j}$. Hence, we probe the system in different coupling regimes and consider all possible flux patterns. The solutions' histograms as well as the calculated means $\mu\left(\log _{10} \lambda_{i}\right)$ and standard deviations $\sigma\left(\log _{10} \lambda_{i}\right)$ are presented in Fig. 10. We find the histograms to be considerably broadened single-peak distributions, indicating relatively stable means for $\log _{10} \lambda_{2}, \log _{10} \lambda_{3}$ with varying means of $\log _{10} \lambda_{1}$ for variable exponents $\varepsilon$.

From the previous section, Sec. III, we concluded that attractively coupled networks $(\varepsilon>1)$ are able to generate robust, reticulated structures for increased coupling rates of $\lambda_{1}$ and are supported in this by increased fluctuation rates $\lambda_{3}$. The
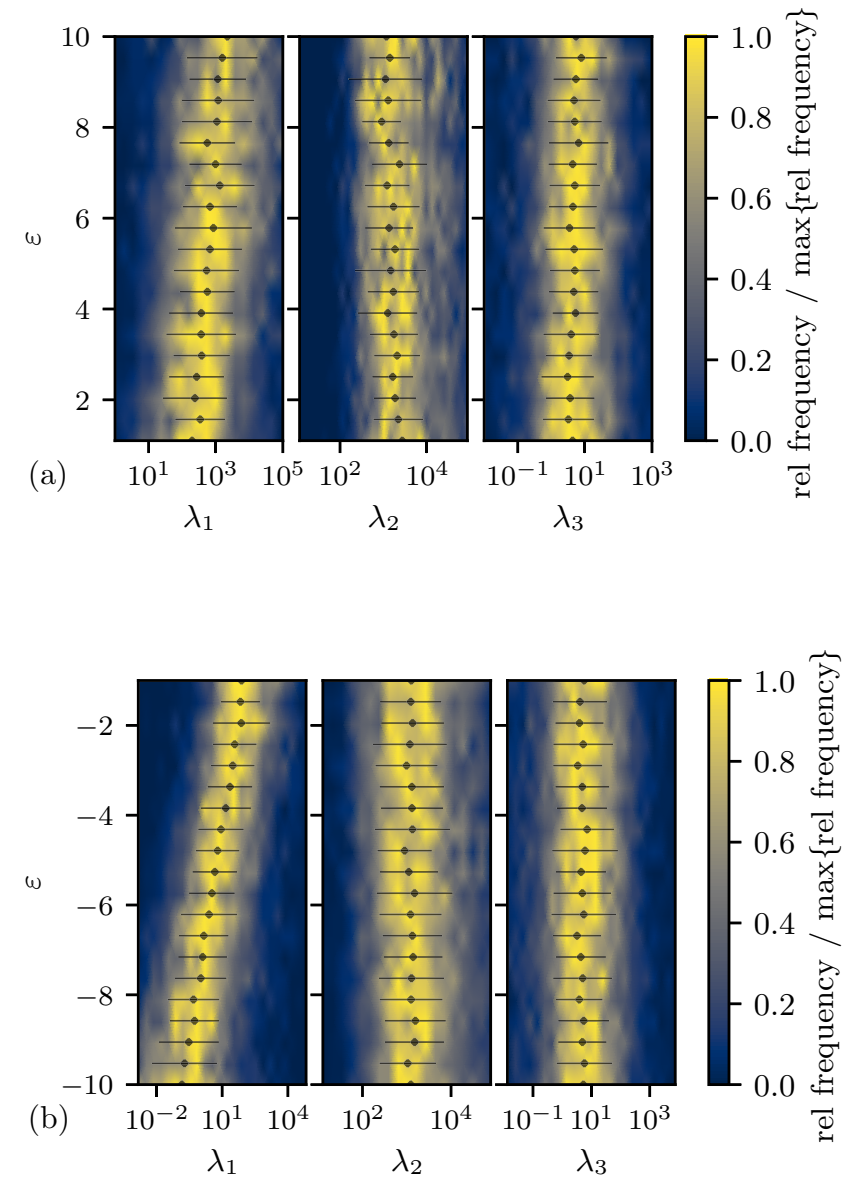

FIG. 10. Estimated interaction parameters for sinusoidal networks indicate fluctuation-dominated nullity state for either coupling interaction: Parameter distributions for the sinusoidal system near the central vein (ROI setting is $R=397 \mu \mathrm{m}, \delta=30 \mu \mathrm{m}$ ) for different coupling exponents $\varepsilon$ representing (a) attractive coupling and (b) repulsive coupling.

estimates for the attractive coupling case in Fig. 10(a) indicate that increased fluctuation rates are present $\left[\mu\left(\log _{10} \lambda_{3}\right)>0\right]$, which may account for reticulated structures in flow-driven adaption. Further, we observe monotonically increasing rates of coupling for increasing $\varepsilon$. Interestingly, we find the coupling rates poised just below the actual onset of the topological transition, e.g., $\mu\left(\log _{10} \lambda_{1}\right) \pm \sigma\left(\log _{10} \lambda_{1}\right)=2.56 \pm 1.04$, for the coupling exponent $\varepsilon=3$.

Further, we find the repulsive coupling case to reproduce the same regime of values for $\log _{10} \lambda_{2}, \log _{10} \lambda_{3}$, indicating reticulation by flow fluctuation. Yet the coupling parameter $\mu\left(\log _{10} \lambda_{1}\right)$ displays a monotonically decreasing behavior for decreasing values of $\varepsilon$; see Fig. 10(b). Those low values of $\log _{10} \lambda_{1}$ suggest repulsive interactions to be negligible as the estimates lie far from the regimes with topological implication, e.g., $\mu\left(\log _{10} \lambda_{1}\right) \pm \sigma\left(\log _{10} \lambda_{1}\right)=1.94 \pm 1.03$ for the coupling exponent $\varepsilon=-1$.

Unfortunately, all estimates $\mu\left(\log _{10} \lambda_{i}\right)$ are accompanied by large standard deviations, which in the logarithmic context span orders of magnitudes. Further, we find no indication for a specific coupling scenario $\varepsilon$, e.g., based on a collapse 
of the standard deviation for a specific $\varepsilon$, parameter distributions contradicting topological structure, etc. We suspect these issues to originate from several sources: segmentation inaccuracies' during image analysis, crude approximation of the sink-source landscape of the system, the chosen algorithm of complexity reduction, and ambiguity of numeric solutions due to the nonlinearity of the problem. Ultimately, the very fact that we only make an educated guess about the adaptation mechanisms might exclude other essential principles of selforganized vessel adaptation in the liver lobule. Nevertheless, considering these findings and the restrictions of our model's approach, we assume the emergence of reticulated sinusoidal structures to be the product of flux fluctuations rather than of the newly proposed geometrical interactions. With this technique, we have shown that it is possible to extract order of magnitude estimates of otherwise inaccessible parameters of real adapting biological networks, and in doing so to make qualitative statements about the relative strength or importance of different feedbacks.

\section{DISCUSSION}

We have shown that spatial coupling presents another potential mechanism of controlling the topological complexity of optimal transport systems in 3D. We considered the special case of "single source and multiple sinks" in combination with simple cubic lattices as plexi as the simplest case possible. In the case of repelling networks, we find this interaction to reduce the networks' relative loop density and provide another method by which a system may be tuned toward its final architecture. It is also possible to retrieve tree-like states at increased fluctuation levels, which represents a stabilization mechanism for spanning trees in noisy networks. Nevertheless, the onset of redundancy is primarily driven by the existence of flow fluctuations. On the other hand, we have shown that attracting vessel surfaces allow for the robust emergence of loops on their own. Even the full recovery of the initial plexus is feasible beyond the coupling-induced nullity transition, in contrast to what is possible with fluctuations. This presents a mechanism which allows for the maintenance of dissipation-minimized reticulated vessel networks as long as there is an effective scaffold providing a positive growth feedback. No qualitative differences could be found in the phase diagrams in comparison with the dual diamond or dual Laves graphs. Our model may be interpreted as a toy model for intertwined flow networks as found in the mammalian liver lobules and other organ structures, such as kidneys, pancreas, or bone marrow. The cost-function ansatz, though, provides a generally applicable tool in network optimization and could be profitably applied to other boundary conditions or graph geometries which resemble realistic structures.

Our approach further enabled us to derive a more general form of Murray's law, directly involving flow interactions and environmental couplings. We find this technique to predict the model parameters with high fidelity for simulated Kirchhoff networks given their topology and respective edge radii distributions. In the same manner, we find order of magnitude estimates for the parameters in experimentally acquired data sets of sinusoids in liver lobule of mice. Hence, one could consider this methodology as an effective classification of spatially adapting network structures allowing us to probe for relevant parameter regimes of adaptation models.

We aim to expand the purely geometric approach, which was studied here, by explicit involvement of hydrodynamicchemical feedback between a vessel and its local environment. This would be of particular concern in intertwined distribution networks transferring water and metabolites with their respective partner network. Biological systems, such as the capillary networks in the liver lobule, present complex dual transport systems where the actual flow rates are not necessarily influenced by their respective partner network's flow rate [52] but rather by the concentration of bile salt components transported [27] as well as secretion rates of hepatocytes. Subsequently, future studies should consider concentration or pump-rate dependent flow rate perturbations in the optimization model. One may also consider a direct postulation of cross-network feedback in the adaptation dynamics to account for the actual network structures. Recent studies regarding solute transport and optimization of cross-wall transport $[53,54]$ might present a suitable basis for such an approach. Eventually, we strive toward a generalized formulation of environmental factors whose influence can be depicted in the form of local adaptation rules of complex flow networks. Ultimately, complex distribution and flow systems that respect and leverage their spatial embeddings remain a rich topic with myriad opportunities both to make contact with applied and biological settings and to open up new ways to understand the physics of complex systems.

\section{ACKNOWLEDGMENTS}

Felix Kramer gratefully acknowledges support from the German Federal Ministry of Education and Research (BMBF), Grant No. 031L0044 (SYSBIO II). Our special thanks go to Fabian Segovia-Miranda for conducting the relevant experimental work and Hernan Morales-Navarrete for the subsequent image reconstruction, as well as for providing us with the sinusoidal and canaliculial skeleton data sets. We would like to thank Marino Zerial, Julien Delpierre, Yannis Kalaidzidis, Quentin Vagne, Dora Tang, Andre Nadler, Mark Warner, and all members of the Modes Lab and Zechner Lab for their comments, helpful discussions, and resourceful feedback throughout the process of creating this work. We would further like to thank the reviewers and editors for their constructive feedback.

\section{APPENDIX A: MINIMIZATION OF CUSTOM COST FUNCTION $\Gamma$}

To minimize the cost function $\Gamma$ as given in Sec. II, we write it as

$$
\begin{gathered}
\Gamma=\Gamma_{12}+\Gamma_{1}+\Gamma_{2} \\
\text { with } \Gamma_{1}=\frac{\pi}{8 \eta_{1} l_{1}} \sum_{e_{1}}\left[\Delta p_{e_{1}}^{2} r_{e_{1}}^{4}+a_{1} r_{e_{1}}^{2}\right] \\
\text { and } \Gamma_{2}=\frac{\pi}{8 \eta_{2} l_{2}} \sum_{e_{2}}\left[\Delta p_{e_{2}}^{2} r_{e_{2}}^{4}+a_{2} r_{e_{2}}^{2}\right] .
\end{gathered}
$$


We may further use the vectorial notation for dissipationvolume terms $\Gamma_{1}, \Gamma_{2}$, using (5), to formulate it in terms of the nodal sinks and/or sources (here just for $\Gamma_{1}$; derivation for $\Gamma_{2}$ is performed analogously),

$$
\begin{aligned}
\Gamma_{1} & =\Delta \boldsymbol{p}^{T} \cdot \boldsymbol{C} \cdot \Delta \boldsymbol{p}+q \operatorname{Tr}\left(\boldsymbol{C}^{1 / 2}\right) \\
& =\boldsymbol{s}^{T}\left[\boldsymbol{B}^{T} \boldsymbol{C B}\right]^{\dagger} \boldsymbol{s}+q \operatorname{Tr}\left(\boldsymbol{C}^{1 / 2}\right)
\end{aligned}
$$

with $q=a_{1}\left(\frac{\pi}{8 \eta_{1} l_{1}}\right)^{1 / 2}$ and $c_{e_{1}}=\frac{\pi r_{e_{1}}^{4}}{8 \eta_{1} l_{1}}$ as entries of the diagonal $\boldsymbol{C}$. We calculate the (pseudo)time derivatives of $P$ to be

$$
\begin{aligned}
\frac{d \Gamma_{1}}{d t}= & \boldsymbol{s}^{T} \partial_{t}\left[\boldsymbol{B} \cdot \boldsymbol{C} \cdot \boldsymbol{B}^{T}\right]^{\dagger} \boldsymbol{s} \\
& +2 \boldsymbol{s}^{T}\left[\boldsymbol{B} \cdot \boldsymbol{C} \cdot \boldsymbol{B}^{T}\right]^{\dagger} \partial_{t} \boldsymbol{s}+q \partial_{t} \operatorname{Tr}\left(\boldsymbol{C}^{1 / 2}\right) .
\end{aligned}
$$

The derivative of the generalized inverse $\boldsymbol{B} \cdot \boldsymbol{C} \cdot \boldsymbol{B}^{T}=\boldsymbol{A}$ is [55]

$$
\begin{aligned}
\partial_{t} \boldsymbol{A}^{\dagger}= & -\boldsymbol{A}^{\dagger}\left(\boldsymbol{B} \cdot \partial_{t} \boldsymbol{C} \cdot \boldsymbol{B}^{T}\right) \boldsymbol{A}^{\dagger} \\
& +\left[\boldsymbol{I}-\boldsymbol{A}^{\dagger} \cdot \boldsymbol{A}\right]\left(\boldsymbol{B} \cdot \partial_{t} \boldsymbol{C} \cdot \boldsymbol{B}^{T}\right) \boldsymbol{A}^{T \dagger} \cdot \boldsymbol{A}^{\dagger} \\
& +\boldsymbol{A}^{\dagger} \cdot \boldsymbol{A}^{T \dagger}\left(\boldsymbol{B}^{T} \cdot \partial_{t} \boldsymbol{C} \cdot \boldsymbol{B}\right)\left[\boldsymbol{I}-\boldsymbol{A} \cdot \boldsymbol{A}^{\dagger}\right]
\end{aligned}
$$

Fortunately, the projector terms vanish as we have

$$
\boldsymbol{s}^{T}\left[\boldsymbol{I}-\boldsymbol{A}^{\dagger} \cdot \boldsymbol{A}\right]=0 \text { and }\left[\boldsymbol{I}-\boldsymbol{A} \cdot \boldsymbol{A}^{\dagger}\right] \boldsymbol{s}=0 .
$$

Together with the identity $\boldsymbol{A}^{\dagger}=\left(\boldsymbol{A}^{T}\right)^{\dagger}$, the total time derivative of $\Gamma_{1}$ becomes

$$
\begin{aligned}
\frac{d \Gamma_{1}}{d t}= & -\boldsymbol{s}^{T} \boldsymbol{A}^{T \dagger}\left(\boldsymbol{B} \cdot \partial_{t} \boldsymbol{C} \cdot \boldsymbol{B}^{T}\right) \boldsymbol{A}^{\dagger} \boldsymbol{s} \\
& +2 \boldsymbol{s}^{T} \boldsymbol{A}^{\dagger} \partial_{t} \boldsymbol{s}+q \partial_{t} \operatorname{Tr}\left(\boldsymbol{C}^{1 / 2}\right) .
\end{aligned}
$$

With partial derivatives, simplifying this formula gives

$$
\begin{gathered}
\partial_{t} \operatorname{Tr}\left(\boldsymbol{C}^{1 / 2}\right)=\frac{1}{2} \operatorname{Tr}\left(\boldsymbol{C}^{-1 / 2} \cdot \partial_{t} \boldsymbol{C}\right), \\
\partial_{t} \boldsymbol{s}=0 .
\end{gathered}
$$

As we also have $\Delta \boldsymbol{p}=\boldsymbol{B}^{T} \cdot \boldsymbol{A}^{\dagger} \boldsymbol{s}$, we may write the total time derivative as

$$
\frac{d \Gamma_{1}}{d t}=-\Delta \boldsymbol{p}^{T} \cdot \partial_{t} \boldsymbol{C} \cdot \Delta \boldsymbol{p}+\frac{q}{2} \operatorname{Tr}\left(\boldsymbol{C}^{-1 / 2} \partial_{t} \boldsymbol{C}\right) .
$$

With diagonals $\partial_{t} c_{e_{1}}=\frac{\pi r_{e_{1}}^{3}}{2 \eta_{1} l_{1}} \partial_{t} r_{e_{1}}$ and resubstituting $q$, this becomes

$$
\frac{d \Gamma_{1}}{d t}=\frac{\pi}{2 \eta_{1} l_{1}} \sum_{e_{1}} r_{e_{1}}\left[\frac{a_{1}}{2}-\Delta p_{e_{1}}^{2} r_{e_{1}}^{2}\right] \partial_{t} r_{e_{1}} .
$$

The coupling component's derivative $\Gamma_{12}$ may be calculated with partial derivatives,

$$
\frac{d \Gamma_{12}}{d t}=-\frac{b \varepsilon}{2}\left[\sum_{e_{1} e_{2}} F_{e_{1} e_{2}} \Delta r_{e_{1} e_{2}}^{\varepsilon-1} \partial_{t} r_{e_{1}}+\sum_{e_{1} e_{2}} F_{e_{1} e_{2}} \Delta r_{e_{1} e_{2}}^{\varepsilon-1} \partial_{t} r_{e_{2}}\right]
$$

so by combining (A13) and (A14) we get, for the total derivative of $\Gamma$,

$$
\begin{aligned}
\frac{d \Gamma}{d t}= & \frac{\pi}{2 \eta_{1} l_{1}} \sum_{e} r_{e_{1}}\left[\frac{a_{1}}{2}-\Delta p_{e_{1}}^{2} r_{e_{1}}^{2}\right] r_{e_{1}} \partial_{t} r_{e_{1}} \\
& +\frac{\pi}{2 \eta_{2} l_{2}} \sum_{e_{2}}\left[\frac{a_{2}}{2}-\Delta p_{e_{2}}^{2} r_{e_{2}}^{2}\right] r_{e_{2}} \partial_{t} r_{e_{2}} \\
& -\frac{b \varepsilon}{2}\left[\sum_{e_{1}} F_{e_{1} e_{2}} \Delta r_{e_{1} e_{2}}^{\varepsilon-1} \partial_{t} r_{e_{1}}+\sum_{e_{2}} F_{e_{1} e_{2}} \Delta r_{e_{1} e_{2}}^{\varepsilon-1} \partial_{t} r_{e_{2}}\right] .
\end{aligned}
$$

To find a local minimum of $\Gamma$ when progressing through (pseudo)time $t$, we have to ensure that $d \Gamma \leqslant 0$. So we may minimize $\Gamma$, with auxiliary functions $\chi_{i} \geqslant 0$ by choosing

$$
\begin{aligned}
& \partial_{t} r_{e_{1}} / \chi_{1}=\left[\Delta p_{e_{1}}^{2} r_{e_{1}}^{2}-\alpha_{1}\right] r_{e_{1}}+\beta_{1} \operatorname{sgn}(\varepsilon) \sum_{e_{2}} F_{e_{1} e_{2}} \Delta r_{e_{1} e_{2}}^{\varepsilon-1}, \\
& \partial_{t} r_{e_{2}} / \chi_{2}=\left[\Delta p_{e_{2}}^{2} r_{e_{2}}^{2}-\alpha_{2}\right] r_{e_{2}}+\beta_{2} \operatorname{sgn}(\varepsilon) \sum_{e_{1}} F_{e_{1} e_{2}} \Delta r_{e_{1} e_{2}}^{\varepsilon-1},
\end{aligned}
$$

where we set $\alpha_{i}=\frac{a_{i}}{2}, \beta_{i}=\frac{b \eta_{i} l_{i}|\varepsilon|}{\pi}$ for compact notation.

\section{APPENDIX B: UNCORRELATED AND COUPLED FLOW FLUCTUATIONS}

In this section, we give a detailed derivation of the analytic form of the mean squared pressure in case of uncorrelated, identically distributed sink fluctuations as introduced in Ref. [14] and discussed in Sec. II.

From the current law (1) in combination with Ohm's law (2), one knows that the sum over all in- and outflows of the system vanishes [56], i.e., $\sum_{v} s_{v}=0$. When considering the sink conditions as well as the source constraint defined in the theory section, we may write the moments as (putting the distinguished source at $v=0$ )

$$
\begin{gathered}
\left\langle s_{v}\right\rangle=\mu, \\
\left\langle s_{0}\right\rangle=-\left\langle\sum_{v>0} s_{v}\right\rangle=(1-n) \mu, \\
\left\langle s_{v} s_{w}\right\rangle=\delta_{v w} \sigma^{2}+\mu^{2} \text { if } v, w>0, \\
\left\langle s_{0}^{2}\right\rangle=\left\langle\sum_{v, w>0} s_{v} s_{w}\right\rangle=(n-1) \sigma^{2}+(n-1)^{2} \mu^{2}, \\
\left\langle s_{0} s_{w}\right\rangle=-\left\langle\sum_{v>0} s_{v} s_{w}\right\rangle=-(n-1) \mu^{2}-\sigma^{2} .
\end{gathered}
$$

Hence, we may calculate the squared-mean pressure by using the auxiliary conductance tensor $\boldsymbol{A}_{v w}^{e}=\boldsymbol{C}_{e e}^{-1}\left[\boldsymbol{B} \cdot \boldsymbol{C}^{1 / 2}\right]_{e v}^{\dagger}\left[\boldsymbol{B} \cdot \boldsymbol{C}^{1 / 2}\right]_{e w}^{\dagger}$ as

$$
\begin{aligned}
\left\langle\Delta p_{e}^{2}\right\rangle & =\sum_{v w} \boldsymbol{A}_{v w}^{e}\left\langle s_{v} s_{w}\right\rangle \\
& =\boldsymbol{A}_{00}^{e}\left\langle s_{0}^{2}\right\rangle+2 \sum_{v>0} \boldsymbol{A}_{v 0}^{e}\left\langle s_{0} s_{v}\right\rangle+\sum_{v, w>0} \boldsymbol{A}_{v w}^{e}\left\langle s_{v} s_{w}\right\rangle
\end{aligned}
$$




$$
\begin{aligned}
= & \boldsymbol{A}_{00}^{e}(n-1)\left[(n-1) \mu^{2}+\sigma^{2}\right] \\
& -2 \sum_{v>0} \boldsymbol{A}_{v 0}^{e}\left[(n-1) \mu^{2}+\sigma^{2}\right] \\
& +\sum_{v, w>0} \boldsymbol{A}_{v w}^{e}\left[\delta_{v w} \sigma^{2}+\mu^{2}\right] \\
= & \sum_{v, w} \boldsymbol{A}_{v w}^{e}(n-1)\left[(n-1) \mu^{2}+\sigma^{2}\right] \delta_{0 v} \delta_{0 w} \\
& -\sum_{v, w} \boldsymbol{A}_{v w}^{e}\left[(n-1) \mu^{2}+\sigma^{2}\right]\left[\left(1-\delta_{0 w}\right) \delta_{0 v}\right] \\
& -\sum_{v, w} \boldsymbol{A}_{v w}^{e}\left[(n-1) \mu^{2}+\sigma^{2}\right]\left[\left(1-\delta_{0 v}\right) \delta_{0 w}\right] \\
& +\sum_{v, w} \boldsymbol{A}_{v w}^{e}\left[\delta_{v w} \sigma^{2}+\mu^{2}\right]\left(1-\delta_{0 v}\right)\left(1-\delta_{0 w}\right) .
\end{aligned}
$$

Ordering the terms for $\mu$ and $\sigma$, respectively, we can acquire the coefficient matrices $\boldsymbol{U}$ and $\boldsymbol{V}$,

$$
\begin{aligned}
\left\langle\Delta p_{e}^{2}\right\rangle= & \sum_{v, w} \boldsymbol{A}_{v w}^{e} \mu^{2}\left\{\left(1-\delta_{0 v}\right)\left(1-\delta_{0 w}\right)+\delta_{0 v} \delta_{0 w}(n-1)^{2}\right. \\
& \left.-(n-1)\left[\delta_{0 v}\left(1-\delta_{0 w}\right)+\delta_{0 w}\left(1-\delta_{0 v}\right)\right]\right\} \\
& +\sum_{v, w} \boldsymbol{A}_{v w}^{e} \sigma^{2}\left[\left(1-\delta_{0 w}\right)\left(1-\delta_{0 v}\right) \delta_{v w}-\delta_{0 v}\left(1-\delta_{0 w}\right)\right. \\
& \left.-\delta_{0 w}\left(1-\delta_{0 v}\right)+(n-1) \delta_{0 v} \delta_{0 w}\right] \\
= & \sum_{v, w} \boldsymbol{A}_{v w}^{e} \mu^{2}\left[1+\delta_{0 v} \delta_{0 w} n^{2}-n\left(\delta_{0 v}+\delta_{0 w}\right)\right] \\
& +\sum_{v, w} \boldsymbol{A}_{v w}^{e} \sigma^{2}\left[\delta_{v w}+\left(n+1+\delta_{v w}\right) \delta_{0 v} \delta_{0 w}\right. \\
& \left.-\left(1+\delta_{v w}\right)\left(\delta_{0 v}+\delta_{0 w}\right)\right] .
\end{aligned}
$$

We further suggest to expand this ansatz by introducing additional sources which act as clones of the very first one; i.e., we will have $s_{p}=s_{q}$ using the indices $p, q$ for sources and $m, n$ for sinks. Then conditions (B2), (B4), and (B5) will become for $a$ sources and $b$ sinks (with $a+b=n$ ),

$$
\begin{aligned}
\left\langle\sum_{p} s_{p}\right\rangle & =-\left\langle\sum_{u} s_{u}\right\rangle=-b \mu \rightarrow\left\langle s_{p}\right\rangle=-\frac{b}{a} \mu, \\
\left\langle s_{p} s_{q}\right\rangle & =\frac{1}{a^{2}}\left\langle\sum_{u, v} s_{u} s_{v}\right\rangle=\frac{1}{a^{2}}\left(b \sigma^{2}+b^{2} \mu^{2}\right), \\
\left\langle s_{p} s_{u}\right\rangle & =-\frac{1}{a}\left\langle\sum_{v} s_{v} s_{u}\right\rangle=-\frac{1}{a}\left(b \mu^{2}-\sigma^{2}\right) .
\end{aligned}
$$

Hence, we may calculate the mean squared pressure and its coefficient matrices respectively as

$$
\begin{aligned}
\left\langle\Delta p_{e}^{2}\right\rangle= & \sum_{v, w} \boldsymbol{A}_{v w}^{e} \mu^{2}\left[\frac{b^{2}}{a^{2}} \sum_{p, q} \delta_{p v} \delta_{q w}\right. \\
& \left.-\frac{b}{a} \sum_{p, m}\left(\delta_{p v} \delta_{m w}+\delta_{m v} \delta_{p w}\right)+\sum_{m, n} \delta_{m v} \delta_{n w}\right]
\end{aligned}
$$
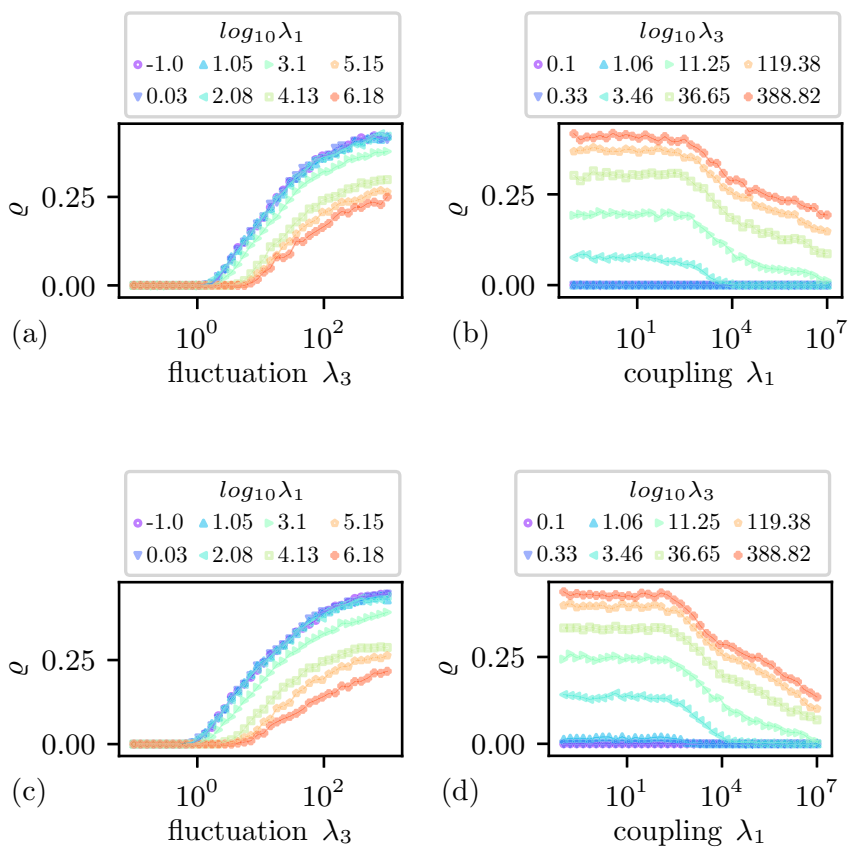

FIG. 11. Nullity $\varrho$ transitions for repulsively coupled networks $(\varepsilon=-1)$ : Symmetric fluctuation $\lambda_{3}$ induced nullity onset and coupling $\lambda_{1}$ induced nullity breakdown for [(a), (b)] network 1 and [(c), (d)] network 2 .

$$
\begin{aligned}
& +\sum_{v, w} \boldsymbol{A}_{v w}^{e} \sigma^{2}\left[\frac{b}{a^{2}} \sum_{p, q} \delta_{p v} \delta_{q w}\right. \\
& \left.-\frac{1}{a} \sum_{p, m}\left(\delta_{p v} \delta_{m w}+\delta_{m v} \delta_{p w}\right)+\sum_{m, n} \delta_{m v} \delta_{n w} \delta_{m n}\right] .
\end{aligned}
$$

\section{APPENDIX C: SIMULATING COUPLED, ADAPTING NETWORKS}

In this section, we show additional material of the simulation results as performed in Sec. III for both of the networks. In Figs. 11 and 12, we display the nullity transition curves for the cases of repulsive and attractive coupling. It may be noted here once again that all simulation parameters $\lambda_{i}$ were initialized identically for the two networks, meaning any deviations in $\varrho$ have to be caused by topological differences. This phenomenon is particularly apparent in the case of attractive coupling in cubic lattices where the nullity transition in the respective networks becomes smeared out differently for the two structures. Repeating the simulations for coupled networks consisting of complementary Laves graphs or diamond lattices, we find this effect negligible. In the case of repulsive coupling [Figs. 13(a) and 13(b)], both networks display a shift of the onset of fluctuation-induced loops as well as a shift of the estimated saturation point. It may be noted though that a change of several orders of magnitude in the coupling $\lambda_{1}$ is necessary in order to shift the fluctuation onset at all. It is nevertheless crucial to note that the overall nullity is reduced even in the saturated case, which itself tends to be achieved only for significantly higher fluctuations $\lambda_{3}$. 

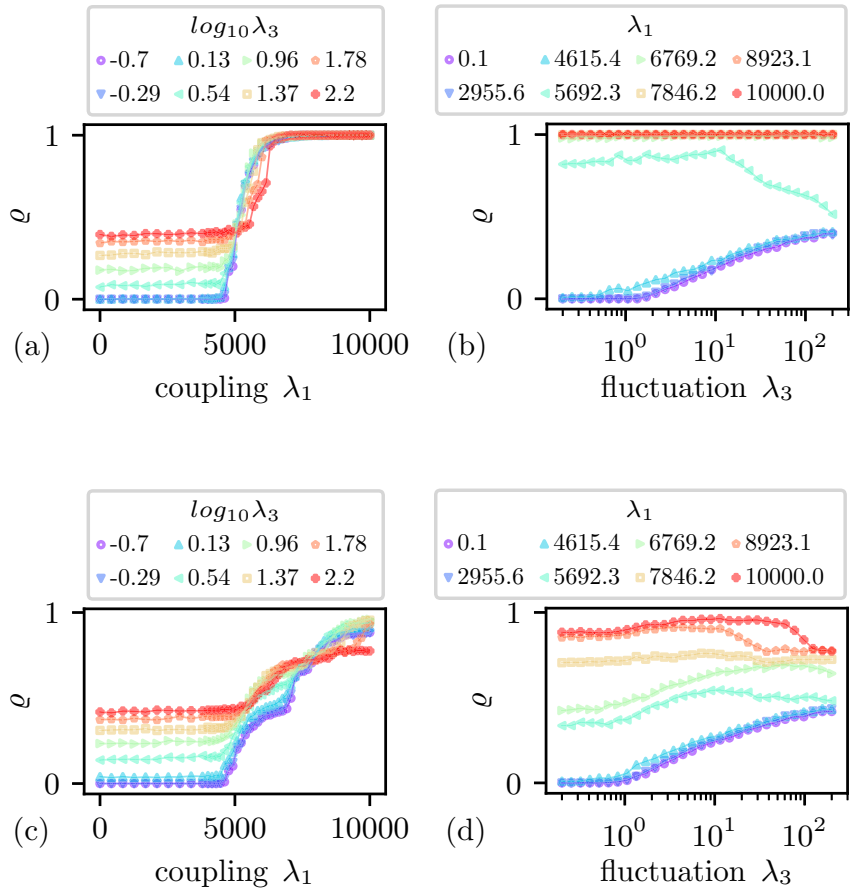

FIG. 12. Nullity $\varrho$ transitions for attractively coupled networks $(\varepsilon=3)$ : Slightly asymmetric coupling $\lambda_{1}$ induced nullity onset and fluctuation $\lambda_{3}$ induced nullity transition for [(a), (b)] network 1 and $[(\mathrm{c}),(\mathrm{d})]$ network 2 .

A similar trend of shifting may be observed in the case of attractive coupling [Figs. 13(c) and 13(d)]. The onset of full plexus recovery as well as the saturation onset develop on similar scales. Note that the trajectories for network 2 suggest the possibility of re-entrant behavior between coupling- and fluctuation-dominated nullity regimes.
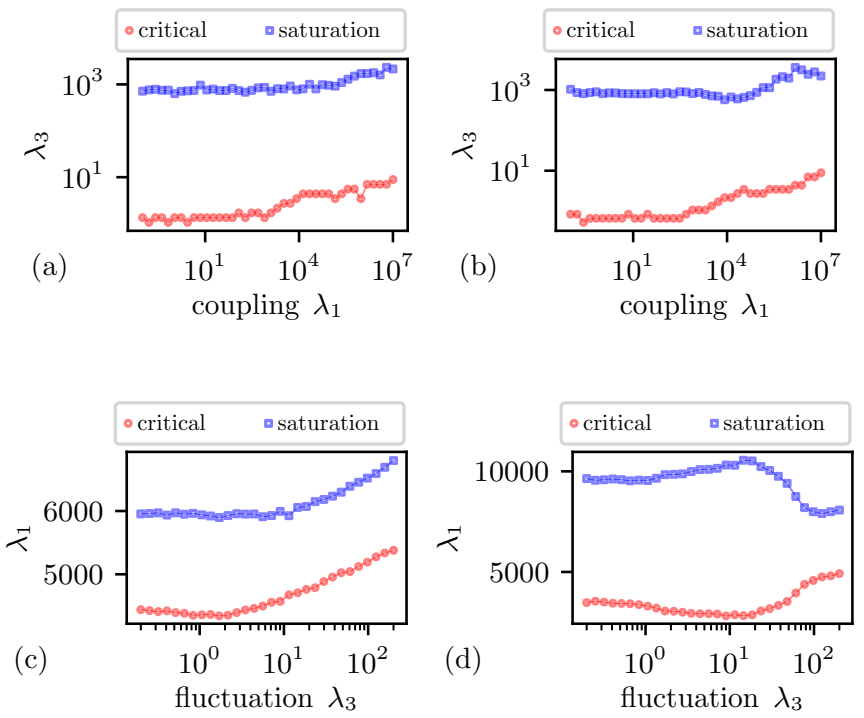

FIG. 13. Shift of onset and saturation points of the fluctuation $\lambda_{3}$ induced nullity transitions for networks 1 and 2 [(a), (b)] for repulsive coupling, here displayed for negative coupling exponent $\varepsilon=-1$. [(c), (d)] Shift of onset and saturation points of the attractive coupling $\lambda_{1}$ induced nullity transitions shift for varying fluctuation $\lambda_{3}$, here displayed for positive coupling exponent $\varepsilon=3$.

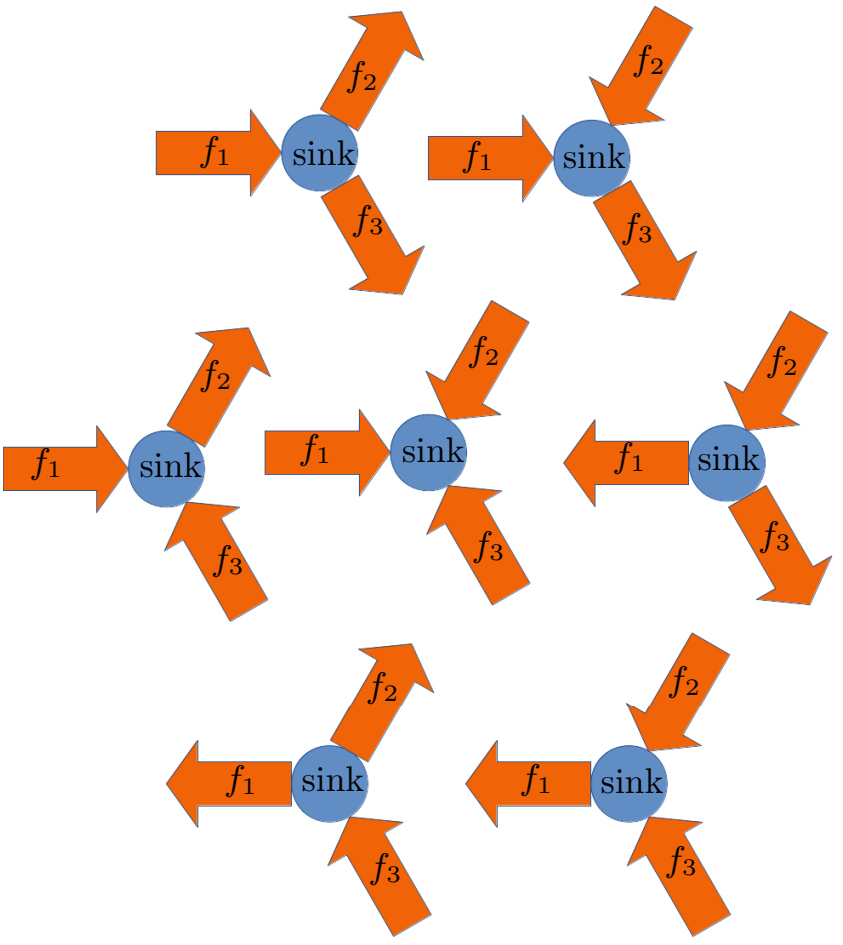

FIG. 14. Scheme of identifying different flow combinations at a sink determining the respective sign of $\Theta_{v e}$, here shown for Ybranching point. Let us identify the sink as $i=0$ and the respective flow carrying edges as $e \in\{1,2,3\}$ and define an incidence triplet $\boldsymbol{\Theta}=\left(\Theta_{01}, \Theta_{02}, \Theta_{03}\right)$. Reading from left to right, we have in the top diagram $\boldsymbol{\Theta}=(1,-1,-1), \boldsymbol{\Theta}=(1,1,-1)$; middle diagram $\boldsymbol{\Theta}=$ $(1,-1,1), \boldsymbol{\Theta}=(1,1,1), \boldsymbol{\Theta}=(-1,1,-1)$; bottom diagram $\boldsymbol{\Theta}=$ $(-1,-1,1), \boldsymbol{\Theta}=(-1,1,1)$.

\section{APPENDIX D: GEOMETRIC LAWS IN COUPLED, NOISY NETWORKS}

This section is focused on the geometric law discussed in Sec. IV. One can show that introducing fluctuations and coupling alter the classical form of Murray's law $r_{0}^{3}=r_{1}^{3}+r_{2}^{3}$ in the following way: Given the Kirchhoff current law and rewriting it via Ohm's law, we get for all sink vertices $i$

$$
\sum_{e \in \operatorname{inc}(v)} f_{e}=s_{v} \Leftrightarrow \sum_{e \in \operatorname{inc}(v)} r_{e}^{* 4} \Delta p_{e}^{*}=s_{v}^{*} .
$$

Taking the average over all pressure configurations between two adaptation events, we get

$$
\sum_{e \in \operatorname{inc}(v)} r_{e}^{* 4}\left\langle\Delta p_{e}^{*}\right\rangle=\left\langle s_{v}^{*}\right\rangle \Leftrightarrow \sum_{e \in \operatorname{inc}(v)} \Theta_{v e} r_{e}^{* 4}\left|\left\langle\Delta p_{e}^{*}\right\rangle\right|=\left\langle s_{v}^{*}\right\rangle
$$

with an effective incidence factor $\Theta_{v e}=\left\{\begin{array}{l} \pm 1 \\ 0\end{array}\right.$ distinguishing between in- and outgoing flows on the relevant edges; see Fig. 14. In order to acquire the cubic form, we substitute $r_{e}^{*}$ 
with the result of the stationary state's equations,

$$
\begin{gathered}
\partial_{t^{*}} r_{e}^{*}=0 \\
\Rightarrow 0=\left(r_{e}^{* 2}\left\langle\Delta p_{e}^{* 2}\right\rangle-\lambda_{2}\right) r_{e}^{*}+\lambda_{1} \operatorname{sgn}(\varepsilon) \sum_{e^{\prime}} F_{e e^{\prime}} \Delta r_{e e^{\prime}}^{*,-\varepsilon} \\
\Leftrightarrow r_{e}^{*}=\frac{\sqrt{\lambda_{2}-\lambda_{1} r_{e}^{*-1} \operatorname{sgn}(\varepsilon) \sum_{e^{\prime}} F_{e e^{\prime}} \Delta r_{e e^{\prime}}^{*, \varepsilon-1}}}{\sqrt{\left\langle\Delta p_{e}^{* 2}\right\rangle}} .
\end{gathered}
$$

Plugging (D5) into (D2) and rewriting $\left|\left\langle\Delta p_{e}^{*}\right\rangle\right|=\sqrt{\left\langle\Delta p_{e}^{*}\right\rangle^{2}}$, $g_{e}^{*}(\varepsilon)=\operatorname{sgn}(\varepsilon) \sum_{e^{\prime}} F_{e e^{\prime}} \Delta r_{e e^{\prime}}^{*, \varepsilon-1}$, we get

$$
\sum_{e \in \operatorname{inc}(v)} \Theta_{v e} \sqrt{\left(\lambda_{2}-\lambda_{1} r_{e}^{*-1} g_{e}^{*}(\varepsilon)\right) \frac{\left\langle\Delta p_{e}^{*}\right\rangle^{2}}{\left\langle\Delta p_{e}^{* 2}\right\rangle}} r_{e}^{* 3}=\left\langle s_{v}^{*}\right\rangle .
$$

We know from Appendix B that $\left\langle\Delta p_{e}^{2}\right\rangle=\sum_{v w} \boldsymbol{A}_{v w}^{e}\left\langle s_{v} s_{w}\right\rangle$ and may also deduce $\left\langle\Delta p_{e}\right\rangle^{2}=\sum_{v w} \boldsymbol{A}_{v w}^{e}\left\langle s_{v}\right\rangle\left\langle s_{w}\right\rangle$. This enables us to calculate the ratio via the covariance,

$$
\begin{aligned}
\frac{\left\langle\Delta p_{e}^{2}\right\rangle}{\left\langle\Delta p_{e}\right\rangle^{2}} & =\frac{\sum_{v w} \boldsymbol{A}_{v w}^{e}\left\langle s_{v} s_{w}\right\rangle}{\sum_{v w} \boldsymbol{A}_{v w}^{e}\left\langle s_{v}\right\rangle\left\langle s_{w}\right\rangle} \\
& =\frac{\sum_{v w} \boldsymbol{A}_{v w}^{e}\left[\left\langle s_{v}\right\rangle\left\langle s_{w}\right\rangle+\left\langle\left(s_{v}-\left\langle s_{v}\right\rangle\right)\left(s_{w}-\left\langle s_{w}\right\rangle\right)\right\rangle\right]}{\sum_{v w} \boldsymbol{A}_{v w}^{e}\left\langle s_{v}\right\rangle\left\langle s_{w}\right\rangle} \\
& =1+\frac{\sum_{v w} \boldsymbol{A}_{v w}^{e}\left\langle\left(s_{v}-\left\langle s_{v}\right\rangle\right)\left(s_{w}-\left\langle s_{w}\right\rangle\right)\right\rangle}{\sum_{v w} \boldsymbol{A}_{v w}^{e}\left\langle s_{v}\right\rangle\left\langle s_{w}\right\rangle}
\end{aligned}
$$

Substituting this into (D6) and having $\frac{\left\langle\Delta p_{e}^{2}\right\rangle}{\left\langle\Delta p_{e}\right\rangle^{2}}=\frac{\left\langle\Delta p_{e}^{* 2}\right\rangle}{\left\langle\Delta p_{e}^{*}\right\rangle^{2}}, H_{e}=$ $\frac{\sum_{v w} \boldsymbol{A}_{v w}^{e}\left\langle\left(s_{v}-\left\langle s_{v}\right\rangle\right)\left(s_{w}-\left\langle s_{w}\right\rangle\right)\right\rangle}{\sum_{v w} \boldsymbol{A}_{v w}^{e}\left\langle s_{v}\right\rangle\left\langle s_{w}\right\rangle}$, we get

$$
\sum_{e \in \operatorname{inc}(v)} a_{e} r_{e}^{* 3}=\left\langle s_{v}^{*}\right\rangle
$$

$$
\text { setting } a_{e}=\Theta_{v e} \sqrt{\frac{\lambda_{2}-\lambda_{1} r_{e}^{*-1} g_{e}^{*}(\varepsilon)}{1+H_{e}}} \text {. }
$$

One may simplify this complex by considering the following: Assume that we have all randomly fluctuating sinks distributed uncorrelated yet identically as in Appendix B, (B11); then we may set $\left\langle s_{v}^{*}\right\rangle=1$ and re-evaluate $H_{e}$. This leads to the equation presented in Sec. IV,

$$
\begin{gathered}
\sum_{\in \operatorname{inc}(v)} a_{e} r_{e}^{* 3}=1 \\
\text { with } a_{e}=\Theta_{v e} \sqrt{\frac{\lambda_{2}-\lambda_{1} r_{e}^{*-1} g_{e}^{*}(\varepsilon)}{1+\lambda_{3} \frac{\delta \phi_{e}}{\phi_{e}}}} .
\end{gathered}
$$

For experimental validation of (D12), it will be necessary to know the networks vessel radii as well as the sink-source pattern (although it may be sufficient to know where the system's source is and to consider every other node as sink). Considering Y branchings of low sink-source value (points of negligible secretion or leakage) such that $\left|a_{e} r_{e}^{*, 3}\right| \gg 1$, further setting the index for the largest vessel to zero (and accordingly increasing for the other vessel pieces at the branching), we may write

$$
a_{0} r_{0}^{* 3}=a_{1} r_{1}^{* 3}+a_{2} r_{2}^{* 3}
$$
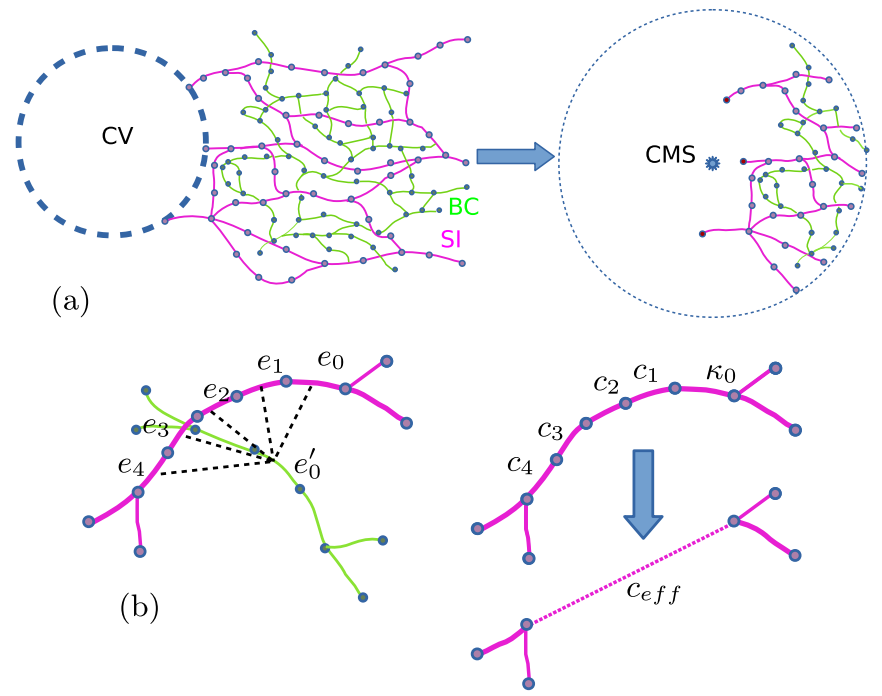

FIG. 15. Setting the range of interest and coarse graining the sinusoidal (SI) and bile canaliculi system (BC): (a) (Left) Vertices closest to the central vein (CV) are identified as sinks. (Right) Determine geometric center of mass of all sinks (CMS) and discard all components, vertices, and edges outside a set perimeter. (b) (Left) Deducting the distance of one path of a network to another path of the partner network by calculating the pairwise distances of all path segments and finding its minimum. (Right) Coarse graining paths into one effective edge, with new edge weight according to addition theorem $c_{\mathrm{eff}}^{*}=\sum_{e} \frac{1}{c_{e}^{*}}$.

In Fig. 15, we show the applied reduction procedure as used in Sec. IV C.

First, the vertices in the sinusoidal network (SI) which are closest to the central vein $(\mathrm{CV})$ are identified. Using these a geometric center of mass (CMS) is calculated and used as the center of a sphere of radius $R$, representing the range of interest (ROI). For any other components, vertices, or edges of any network positioned ROI, see Fig. 15(a). Next, all branching points in the sinusoidal network are identified and all paths $\boldsymbol{p}=\left(e_{i}, \ldots, e_{j}\right)$ consisting of edges $e_{i}$ which start from these points. We proceed for the canaliculi in the same way and then check for each segment of a path $\boldsymbol{p}$ whether there is another segment of another network's path $\boldsymbol{p}^{\prime}$ inside a perimeter $\delta$. If so, these paths count as affiliated. Then we merge all edges

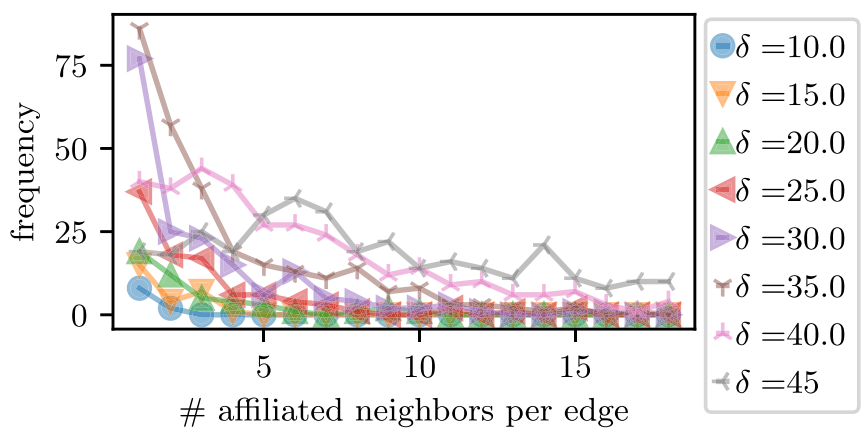

FIG. 16. Histogram displaying the number of affiliated neighbors (edges) found for a given radius around a vessel (threshold, all values the inset in $\mu \mathrm{m}$ ). Total number of pairings found overall for given threshold in the inset for set range of interest $R=397 \mu \mathrm{m}$. 

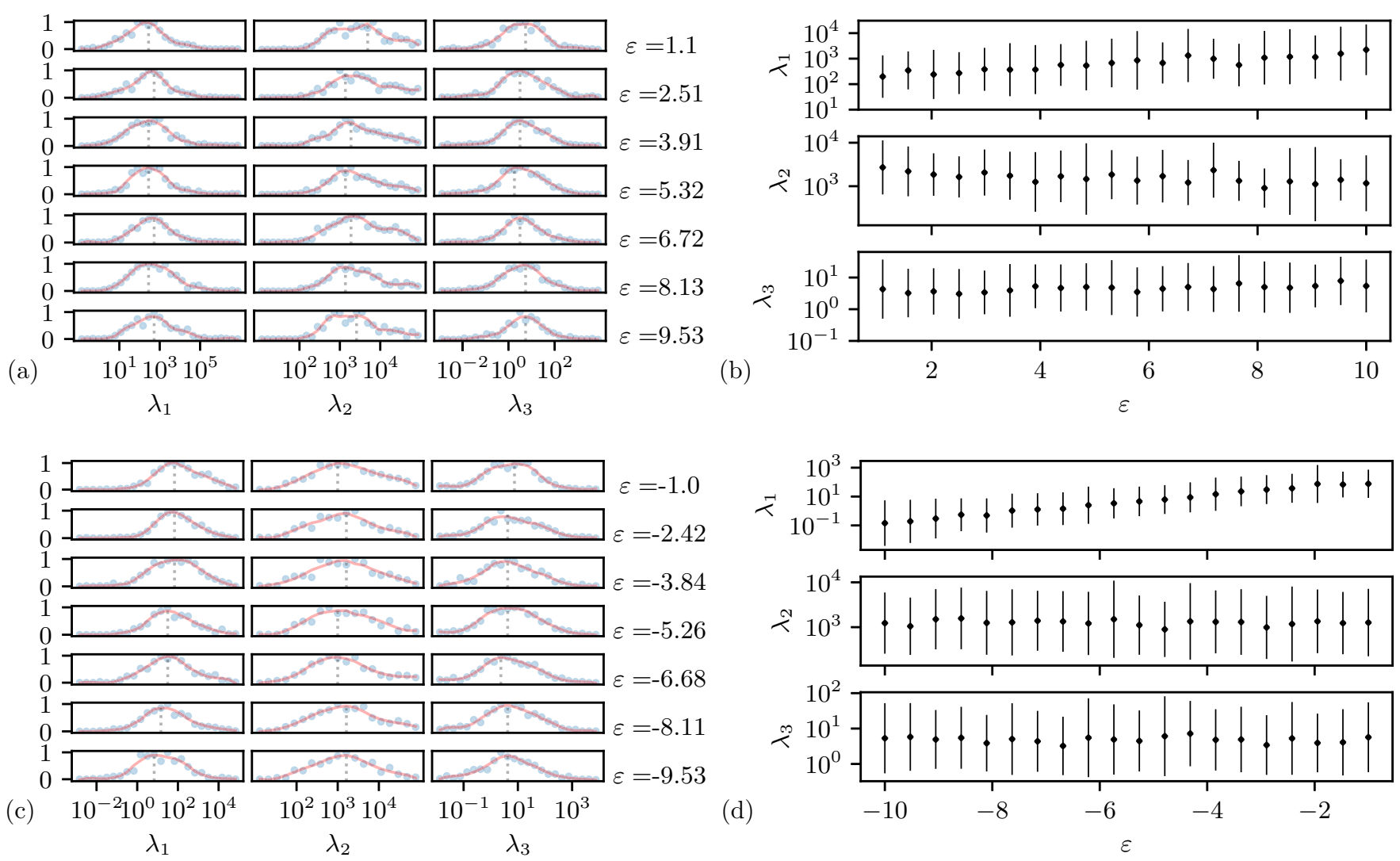

FIG. 17. Estimated parameter distributions with rescaled axis $\log _{10} \lambda_{i}$ (in blue) for selected $\varepsilon$ in the sinusoidal system for $R=397 \mu \mathrm{m}$, $\delta=30 \mu \mathrm{m}$. We applied a Savitzky-Golay regression (in red) to enable an effective peak identification. Subsequently, Gaussian fits are used to calculate the means and standard deviations of the smoothed distributions. [(a), (b)] Attractive coupling. [(c), (d)] Repulsive coupling.

along a path toward a single edge by using the conventional addition theorems for series of resistors, as $c_{\text {eff }}=\sum_{e} \frac{1}{c_{e}}$; see Fig. 15(b). When confronted with data sets of real spatial transport networks such as blood capillaries and secretion channels in Sec. IV C, it becomes important to note that the chosen ROI, namely the radius $R$ and the affiliation perimeter $\delta$, will affect the estimation of the parameters $\log _{10} \lambda_{i}$. For example (see Fig. 16), increasing the affiliation parameter $\delta$ will naturally increase the number of edges affiliated with each other, possibly linking vessel structures of several consecutive neighborhoods.
Further, we attempt to screen through the range of potential coupling exponents $\varepsilon$ in order to find the $\lambda_{i}$ distributions as displayed in Fig. 10, Sec. IV C. In Fig. 17, we display the explicit distributions for selected $\varepsilon$ depicting the different coupling regimes. We smooth the data sets with a SavitzkyGolay filter (red fit in 17) and identify the local maxima which display a prominence of at least $10 \%$ of the maximal function value. We subsequently use Gaussian fits at the identified peak's positions to acquire the mean values and standard deviations as depicted in Figs. 17(b) and 17(d).
[1] F. le Noble, V. Fleury, A. Pries, P. Corvol, A. Eichmann, and R. Reneman, Control of arterial branching morphogenesis in embryogenesis: Go with the flow, Cardiovasc. Res. 65, 619 (2005).

[2] D. Hu, D. Cai, and A. V. Rangan, Blood vessel adaptation with fluctuations in capillary flow distribution, PLoS ONE 7, e45444 (2012).

[3] H. Ronellenfitsch and E. Katifori, Phenotypes of Vascular Flow Networks, Phys. Rev. Lett. 123, 248101 (2019).

[4] W. Risau, Mechanisms of angiogenesis, Nature (London) 386, 671 (1997).

[5] P. Dimitrov and S. W. Zucker, A constant production hypothesis guides leaf venation patterning, Proc. Natl. Acad. Sci. USA 103, 9363 (2006).
[6] T.-H. Nguyen, A. Eichmann, F. Le Noble, and V. Fleury, Dynamics of vascular branching morphogenesis: The effect of blood and tissue flow, Phys. Rev. E 73, 061907 (2006).

[7] A. Lenard, S. Daetwyler, C. Betz, E. Ellertsdottir, H.-G. Belting, J. Huisken, and M. Affolter, Endothelial cell selffusion during vascular pruning, PLoS Biol. 13, e1002126 (2015).

[8] A. Roth-Nebelsick, D. Uhl, V. Mosbrugger, and H. Kerp, Evolution and function of leaf venation architecture: A review, Ann. Bot. 87, 553 (2001).

[9] A. Tero, R. Kobayashi, and T. Nakagaki, A mathematical model for adaptive transport network in path finding by true slime mold, J. Theor. Biol. 244, 553 (2007). 
[10] C. D. Murray, The physiological principle of minimum work I. The vascular system and the cost of blood volume, Proc. Natl. Acad. Sci. USA 12, 207 (1926).

[11] C. D. Murray, The physiological principle of minimum work. II. Oxygen exchange in capillaries, Proc. Natl. Acad. Sci. USA 12, 299 (1926).

[12] S. Bohn and M. O. Magnasco, Structure, Scaling, and Phase Transition in the Optimal Transport Network, Phys. Rev. Lett. 98, 088702 (2007).

[13] E. Katifori, G. J. Szöllősi, and M. O. Magnasco, Damage and Fluctuations Induce Loops in Optimal Transport Networks, Phys. Rev. Lett. 104, 048704 (2010).

[14] F. Corson, Fluctuations and Redundancy in Optimal Transport Networks, Phys. Rev. Lett. 104, 048703 (2010).

[15] A. R. Pries, T. W. Secomb, and P. Gaehtgens, Structural adaptation and stability of microvascular networks: Theory and simulations, Am. J. Physiol.: Heart Circ. Physiol. 275, H349 (1998).

[16] A. R. Pries, B. Reglin, and T. W. Secomb, Structural adaptation of vascular networks, Hypertension 38, 1476 (2001).

[17] T. W. Secomb, J. P. Alberding, R. Hsu, M. W. Dewhirst, and A. R. Pries, Angiogenesis: An adaptive dynamic biological patterning problem, PLoS Comput. Biol. 9, e1002983 (2013).

[18] D. Hu and D. Cai, Adaptation and Optimization of Biological Transport Networks, Phys. Rev. Lett. 111, 138701 (2013).

[19] S.-S. Chang and M. Roper, Microvscular networks with uniform flow., J. Theor. Biol. 462, 48 (2019).

[20] J. Gräwer, C. D. Modes, M. O. Magnasco, and E. Katifori, Structural self-assembly and avalanchelike dynamics in locally adaptive networks, Phys. Rev. E 92, 012801 (2015).

[21] H. Ronellenfitsch and E. Katifori, Global Optimization, Local Adaptation, and the Role of Growth in Distribution Networks, Phys. Rev. Lett. 117, 138301 (2016).

[22] J. Haskovec, P. Markowich, and B. Perthame, Mathematical analysis of a PDE system for biological network formation, Commun. Partial Differ. Eq. 40, 918 (2015).

[23] J. Haskovec, P. Markowich, B. Perthame, and M. Schlottbom, Notes on a PDE system for biological network formation, Nonlinear Anal. 138, 127 (2016).

[24] G. B. West, J. H. Brown, and B. J. Enquist, A general model for the origin of allometric scaling laws in biology, Science 276, 122 (1997).

[25] T. F. Sherman, On connecting large vessels to small: The meaning of Murray's law, J. Gen. Physiol. 78, 431 (1981).

[26] W. W. Lautt, Regulatory processes interacting to maintain hepatic blood flow constancy: Vascular compliance, hepatic arterial buffer response, hepatorenal reflex, liver regeneration, escape from vasoconstriction, Hepatol. Res. 37, 891 (2007).

[27] J. L. Boyer, Bile formation and secretion, Comprehensive Physiology 3, 1035 (2013).

[28] K. Meyer, O. Ostrenko, G. Bourantas, H. Morales-Navarrete, N. Porat-Shliom, F. Segovia-Miranda, H. Nonaka, A. Ghaemi, J.-M. Verbavatz, L. Brusch, I. Sbalzarini, Y. Kalaidzidis, R. Weigert, and M. Zerial, A predictive 3d multi-scale model of biliary fluid dynamics in the liver lobule, Cell Syst. 4, 277 (2017).

[29] H. Morales-Navarrete, F. Segovia-Miranda, P. Klukowski, K. Meyer, H. Nonaka, G. Marsico, M. Chernykh, A. Kalaidzidis, M. Zerial, and Y. Kalaidzidis, A versatile pipeline for the multi- scale digital reconstruction and quantitative analysis of $3 \mathrm{~d}$ tissue architecture, Elife 4, e11214 (2015).

[30] M. M. Shah, R. V. Sampogna, H. Sakurai, K. T. Bush, and S. K. Nigam, Branching morphogenesis and kidney disease, Development 131, 1449 (2004).

[31] F. C. Serluca, I. A. Drummond, and M. C. Fishman, Endothelial signaling in kidney morphogenesis: A role for hemodynamic forces, Curr. Biol. 12, 492 (2002).

[32] J. Magenheim, O. Ilovich, A. Lazarus, A. Klochendler, O. Ziv, R. Werman, A. Hija, O. Cleaver, E. Mishani, E. Keshet, and Y. Dor, Blood vessels restrain pancreas branching, differentiation, and growth, Development 138, 4743 (2011).

[33] A. Villasenor and O. Cleaver, Crosstalk between the developing pancreas and its blood vessels: An evolving dialog, Semin. Cell Dev. Biol. 23, 685 (2012).

[34] D. B. Azizoglu, D. C. Chong, A. Villasenor, J. Magenheim, D. M. Barry, S. Lee, L. Marty-Santos, S. Fu, Y. Dor, and O. Cleaver, Vascular development in the vertebrate pancreas, Dev. Biol. 420, 67 (2016).

[35] L. Planas-Paz and E. Lammert, Mechanosensing in developing lymphatic vessels, in Developmental Aspects of the Lymphatic Vascular System (Springer, Vienna, 2013), pp. 23-40.

[36] K. K. Sivaraj and R. H. Adams, Blood vessel formation and function in bone, Development 143, 2706 (2016).

[37] C. D. Modes, M. O. Magnasco, and E. Katifori, Extracting Hidden Hierarchies in 3D Distribution Networks, Phys. Rev. X 6, 031009 (2016).

[38] L. Papadopoulos, P. Blinder, H. Ronellenfitsch, F. Klimm, E. Katifori, D. Kleinfeld, and D. S. Bassett, Comparing two classes of biological distribution systems using network analysis, PLoS Comput. Biol. 14, e1006428 (2018).

[39] D. Akita, I. Kunita, M. D. Fricker, S. Kuroda, K. Sato, and T. Nakagaki, Experimental models for Murray's law, J. Phys. D: Appl. Phys. 50, 024001 (2016).

[40] R. Penrose, A Generalized Inverse for Matrices (Cambridge University Press, Cambridge, UK, 1955), p. 406-413.

[41] F. P. Kelly, D. R. Cox, and D. M. Titterington, Network routing, Philos. Trans. R. Soc. London, Ser. A 337, 343 (1991).

[42] L. J. Grady and J. R. Polimeni, Discrete Calculus, Applied Analysis on Graphs for Computational Science (Springer Science \& Business Media, Berlin, 2010).

[43] A. Ben-Israel and A. Charnes, Generalized inverses and the Bott-Duffin network analysis, J. Math. Anal. Appl. 7, 428 (1963).

[44] R. Penrose, On Best Approximate Solutions of Linear Matrix Equations (Cambridge University Press, Cambridge, UK, 1956), pp. 17-19.

[45] C. A. Desoer and E. S. Kuh, Basic Circuit Theory (McGrawHill, New York, 1969).

[46] L. D. Landau and E. M. Lifshitz, Fluid Mechanics, Course of Theoretical Physics Vol. 6, 2nd ed. (Elsevier, ButterworthHeinemann, Amsterdam, 2012).

[47] H. Whitney, Non-separable and planar graphs, Trans. Am. Math. Soc. 34, 339 (1932).

[48] W. T. Góźdź and R. Hołyst, Triply periodic surfaces and multiply continuous structures from the Landau model of microemulsions, Phys. Rev. E 54, 5012 (1996).

[49] H. Morales-Navarrete, H. Nonaka, A. Scholich, F. SegoviaMiranda, W. de Back, K. Meyer, R. L. Bogorad, V. Koteliansky, 
L. Brusch, Y. Kalaidzidis, F. Jülicher, B. M. Friedrich, and M. Zerial, Liquid-crystal organization of liver tissue, Elife 8, 1035 (2019).

[50] H. Morales-Navarrete, H. Nonaka, F. Segovia-Miranda, M. Zerial, and Y. Kalaidzidis, Automatic recognition and characterization of different non-parenchymal cells in liver tissue, in 2016 IEEE 13th International Symposium on Biomedical Imaging (ISBI) (IEEE, Prague, 2016), pp. 536-540.

[51] P. Virtanen, R. Gommers, T. E. Oliphant, M. Haberland, T. Reddy, D. Cournapeau, E. Burovski, P. Peterson, W. Weckesser, J. Bright, S. J. van der Walt, M. Brett, J. Wilson, K. J. Millman, N. Mayorov, A. R. J. Nelson, E. Jones, R. Kern, E. Larson, C. J. Carey, I. Polat, Y. Feng, E. W. Moore, J. VanderPlas, D. Laxalde, J. Perktold, R. Cimrman, I. Henriksen, E. A. Quintero, C. R. Harris, A. M. Archibald, A. H. Ribeiro, F. Pedregosa, P. van Mulbregt, and SciPy 1.0 Contributors, SciPy 1.0:
Fundamental Algorithms for Scientific Computing in Python, Nat. Methods 17, 261 (2020).

[52] R. W. Brauer, G. F. Leong, and R. J. Holloway, Mechanics of bile secretion: Effect of perfusion pressure and temperature on bile flow and bile secretion pressure, Am. J. Physiol. 177, 103 (1954).

[53] F. J. Meigel and K. Alim, Flow rate of transport network controls uniform metabolite supply to tissue, J. R. Soc., Interface 15, 20180075 (2018).

[54] O. Ostrenko, J. Hampe, and L. Brusch, Wet-tip versus dry-tip regimes of osmotically driven fluid flow, Sci. Rep. 9, 4528 (2019).

[55] G. H. Golub and V. Pereyra, The differentiation of pseudoinverses and nonlinear least squares problems whose variables separate, SIAM J. Numer. Anal. 10, 413 (1973).

[56] R. J. Duffin, Distributed and lumped networks, J. Math. Mech. 8, 793 (1959) 